\title{
Contact-Line Behavior in Boiling on A Heterogeneous Surface: Physical Insights from Diffuse-Interface Modeling
}

Biao Shen ${ }^{1 *}$, Jiewei Liu², Gustav Amberg², Minh Do-Quang², Junichiro Shiomi ${ }^{3}$, Koji Takahashi ${ }^{1,4}$, Yasuyuki Takata ${ }^{1,5}$

${ }^{1}$ International Institute for Carbon-Neutral Energy Research (WPI-I2CNER), Kyushu University, Motooka 744, Fukuoka 819-3905, Japan

${ }^{2}$ Department of Mechanics, The Royal Institute of Technology, S-100 44 Stockholm, Sweden

${ }^{3}$ Department of Mechanical Engineering, The University of Tokyo, Hongo 7-3-1, Tokyo 113-8656, Japan

${ }^{4}$ Department of Aeronautics and Astronautics, Kyushu University, Motooka 744, Fukuoka 819-3905, Japan

${ }^{5}$ Department of Mechanical Engineering, Kyushu University, Motooka 744, Fukuoka 819-3905, Japan

\begin{abstract}
Enhancement of boiling heat transfer on biphilic (mixed-wettability) surfaces faces a sudden reversal at low pressures, which is brought about by excessive contactline spreading across the wetting heterogeneities. We employ the diffuse-interface approach to numerically study bubble expansion on a heating surface that consists of opposing wettabilities. The results show a dramatic shift in the dynamics of traversing contact line across the wettability divide under different gravities, which correspond to variable bubble growth rates. Specifically, it is found that the contact-line propagation tends to follow closely the rapidly expanding bubble at low gravity, with only a brief interruption at the border between the hydrophobic and hydrophilic sections of the surface. Only when the bubble growth becomes sufficiently weakened at high gravity does the contact line get slowed down drastically to the point of being
\end{abstract}

\footnotetext{
* Corresponding author, TEL (FAX): +8192-802-6749; EMAIL: shen@mech.kyushu-u.ac.jp
} 
nearly immobilized at the edge of the hydrophilic surface. The following bubble expansion, which faces strong limitations in the direction parallel to the surface, features a consistent apparent contact angle around $66.4^{\circ}$, regardless of the wettability combination. A simple theoretical model based on the force balance analysis is proposed to describe the physical mechanism behind such a dramatic transition in the contact-line behavior. 


\section{INTRODUCTION}

More than just an everyday experience, boiling offers one of the most efficient heat transfer solutions[1-3] to various industrial challenges ranging from nuclear reactor cooling $[4,5]$ to thermal management of data centers[6,7], because it capitalizes on the vast reservoir of latent heat of vaporization as well as sensible heat. Dating back to the 1930s when Nukiyama[8] first discovered the so-called boiling curve, the modern research on boiling phenomena - which are inherently multiphase and multiscale[9] and fraught with intractable randomness[10] - continues to garner considerable attention beyond the conventional field of thermal engineering and has fascinated generations of scientists in the intervening years[11]. Pioneering studies by Han and Griffith[12], Mikic et al.[13], Zuber[14,15], and van Stralen et al.[16] all contributed to the classic theory about the pool boiling process: (i) isolated bubbles emerge from pre-existing cavities or defects in the solid surface; (ii) mostly driven by evaporation occurring around and underneath the bubbles (namely, that of a thin microlayer), the bubbles continues to expand under a thermally controlled regime until they turn large enough to be ejected from the surface by growing buoyancy; (iv) heat transfer mechanisms such as microconvection (namely, agitation effect), microlayer evaporation during bubble growth, and regeneration of the superheated liquid layer that is periodically stripped away by the departing bubbles are believed to be responsible for the enhanced heat transport, and (v) the transition to film boiling (i.e., the critical heat flux (CHF)) is mostly dictated by Helmholtz-Taylor instability, which triggers cascading merging of neighboring vapor jetflows and leads eventually to a precipitous decline in the heat transfer efficiency and cataclysmic temperature upsurges.

However, this somewhat simplified physical depiction is now facing mounting challenges from recent advances in precise measurement and engineering innovations in boiling surface design and fabrication that defy conventional thinking. New findings such as the apparently strong dependence of the onset of nucleate boiling (ONB) on surface wettability[17] and the unusually early activation of nucleation sites at very low surface superheats on an ultra-smooth (with a nanoscale roughness) surface[18] 
call into question the validity of the vapor-trapping-cavity theory for bubble nucleation in heterogeneous boiling. An unusually stable presence of dissolved gas near the solid surface[19,20] in the form of surface nanobubbles[21] has been proposed as a plausible alternative nucleation mechanism. Moreover, the significant CHF enhancement achieved through use of nanofluids[22], nanowires[23], microporous coatings[24], microchannels[25], and honeycomb structures[26] has upended the classic hydrodynamic interpretation of the boiling crisis, which is notably devoid of any inputs from the solid side. More accurate predictions of CHF have been made possible by taking into account several previously overlooked surface characteristics including surface wettability change (due to nanoparticle deposition)[27], contact angle hysteresis of the surface[28], capillary wicking of the microstructure[29], dry-out in the porous structure[30], and capillary rewetting of the nanotexture[31].

Emerging from those developments in the understanding of boiling heat transfer is a consensus on the important role of active control of dynamic three-phase contact line. Preferred boiling conditions such as robust nucleate boiling with an effectively delayed CHF can be realized on highly hydrophobic surfaces but with a low receding contact angle (which represents essentially an 'underwater' Wenzel state). Techniques such as purging the surface of noncondensables[32], para-hydrophobic texturing[33], rapid pressurization[34], or electrowetting[35] have been demonstrated to give rise to a Cassie-to-Wenzel transition of the initial wetting state, whereby the minimized surface dewetting is effective in preventing excessive vapor spreading, particularly at high surface heat fluxes. Strong pinning of the contact lines on $b i$-philic (that is, with alternating wettabilities)[20,36] or bi-conductive (namely, endowed with in-plane variations of thermal conductivity)[37] surfaces can bring about ordered bubble growth with separate pathways for escaping vapor from and replenishing liquid to the surface, which was thought to underpin the exceptionally high heat transfer rates seen on micro-channeled surfaces[38]. Such an enhanced state of boiling, however, has been found to be particularly vulnerable to external perturbations such as pressure variations. Our previous study[39] of sub-atmospheric boiling on a biphilic copper 
surface electroplated with an array of hydrophobic Ni-TFEO (tetrafluoroethylene oligomer) spots revealed a sudden transition from continuous boiling to a deleterious mode of intermittent boiling when the pressure dropped sufficiently low. It was further argued[40] that the lateral depinning of the bubble contact lines from the interface between the hydrophobic and hydrophilic surfaces during bubble growth and the resulting 'flooding' of the hydrophobic spots in the wake of bubble departure were responsible for the temporary deactivation of the nucleation sites, which can, in turn, result in a sharp deterioration of boiling heat transfer. Hence, it will be of particular interest to elucidate the mechanism of contact-line depinning on biphilic surfaces, which can possibly lead to an enhanced surface design that is immune to intermittent boiling-induced heat transport deterioration at all pressure levels.

The pinning/depinning behavior of contact line has long attracted the attention of hydrodynamicists studying evaporation of a sessile droplet sitting on a periodic wettability-patterned surface, which shows a strong dependence on the size of the local inhomogeneities[41-43]. It was noted in particular that when crossing the boundary between the hydrophobic and hydrophilic nanotextures, the contact-line dynamics seems to undergo a 'jump' event[41,42] in addition to the famed "stick-slip" (staggered) motion induced by the wetting hysteresis[44]. This intriguing behavior was interpreted to be caused by a special form of contact-line breaking[45].

In contrast, the three-phase contact line is expected to travel at a considerably faster speed in boiling (even reaching the order of $10^{-1} \mathrm{~m} / \mathrm{s}$ on nano-enhanced surfaces[46]) due to the apparently more intense phase change. It will thus be reasonable to postulate that a commensurably stronger energy barrier is required at the surface heterogeneities so as to immobilize or slow down the rapid contact-line motion[42]. Indeed, tantalizing evidence can be found in previous experimental results[40] that the depinning of the bubble contact-line and the following rewetting of the hydrophobic spots tends to occur only at extremely low pressures when the pinning force can no longer hold back the considerably accelerated bubble growth. The aim of the present study is to characterize the three-phase contact line interaction with a 
wettability-patterned surface in a single-component non-isothermal system, which mimics boiling on a biphilic surface. We investigate the contact-line dynamics under the influences of both the bubble growth rate and the local wettability contrast using numerical simulation, which is based on the diffuse-interface method[47]. As a matter of fact, a similar model for a dilute binary mixture of water and nitrogen has been employed to investigate the effect of dissolved gas on the growth and departure of bubbles on biphilic surfaces[20,48].

The remainder of the paper is organized as follows. In Sec. II we describe the diffuse-interface model along with the computational steps. Section III is devoted to the typical numerical results, which show contrasting contact-line spreading behaviors on the biphilic surface under various bubble growth rates. Based on the simulations, we set out to quantify the contact-line dynamics under different combinations of wetting heterogeneities in Sec. IV, which shows, among other things, that while a depinned contact line tends to behave differently in accordance with the local wetting characteristics, several universal features seem to emerge once the contact-line gets pinned at the wettability divide. We further discuss the physical mechanism and critical condition for contact-line pinning on a biphilic surface in Sec. V. Finally, a summary of the results and a few concluding remarks are given in Sec. VI.

\section{NUMERICAL SIMULATION}

Modeling of two-phase flows centers on the treatment of the liquid-vapor interface, which carries extra complexity when mass transfer due to phase change has to be taken into account[49]. The historical debate regarding the nature of the interface can be traced back to the times of Lord Rayleigh[50] and van der Waals[51]. The classic continuum hydrodynamic perspective considers the interface between two immiscible fluids to be a free boundary of zero thickness, where physical quantities exhibit discontinuities between the liquid and vapor phases[47]. Tracking of the interface relies on solving an auxiliary advection equation (notable examples of which include the level-set method[52] and the volume-of-fluid method[53]). Also, effects of capillarity 
need to be explicitly taken into account at the interface. One of the major theoretical flaws of the sharp-interface approach lies with the inherent inconsistency between a moving contact line and the no-slip boundary condition under the Navier-Stokes framework[54]. Although the resulting paradox of unbounded viscous dissipation at the contact line can be remedied by a localized slip model based on the lubrication or thin-film approximation, additional efforts, however, are usually required to match the inner-region solution with that of the outer-region[55].

The alternative approach is to assign a finite width to the interface, which is closer to physical reality especially near the liquid-vapor critical point. Under the diffuse-interface assumption, surface energy (tension) can be derived 'organically' from the smooth transition of density between the two phases. More importantly, the viscous stress singularity that plagues the sharp-interface description can be easily resolved as slip behavior is built in the local equilibrium in the contact-line region[56], which makes the diffuse-interface model a very useful tool to probe two-phase phenomena involving dynamic wetting[57]. For these reasons, we choose to employ the diffuse-interface model based on the dynamic van der Waals theory in an attempt to quantify the contact-line dynamics of a growing bubble on a wettability-patterned surface. That is despite the stringent time- and length-scale limitations and the significantly increased computational complexity, which the method is known to entail[48,58,59].

\section{A. Mathematical formulation}

We consider a two-phase single-component fluid occupying a volume $\Omega$, where the extra cost of free energy associated with the formation of a diffuse liquid-vapor interface can be quantized, to a first approximation[60], by the extended Helmholtz free energy functional,

$$
F=\int_{\Omega} d V\left[f(\rho, T)+\frac{\kappa}{2}|\nabla \rho|^{2}\right]
$$

The first term on the right-hand side represents the bulk contribution (i.e., the classic Helmholtz free energy density), which for a van der Waals fluid is written as 


$$
f(\rho, T)=k_{B} T N_{V}(\rho)\left\{\ln \left[\frac{N_{V}(\rho) T^{3 / 2}}{1-b N_{V}(\rho)}\right]-1\right\}-a N_{V}(\rho)^{2} .
$$

Here the number density is defined as $N_{V}(\rho)=\rho / m_{p}$ (with $m_{p}$ being the molecular mass), $k_{B}$ is the Boltzmann constant, $a$ and $b$ are the molecular parameters in the van der Waals equation of state, and $T$ is temperature.

On the other hand, the second right-hand-side term of square-gradient density $\varrho$ of Eq. (1) denotes the energy excess in the interfacial region. Assuming the gradient energy coefficient $x=$ const[60], we can infer the interfacial thickness from the interpretation of the surface tension (energy) $\sigma$ as the energy cost in creating a smooth liquid-vapor interface,

$$
\sigma=\kappa \int_{\rho_{v}}^{\rho_{l}}\left(\frac{d \rho}{d x}\right)^{2} d x
$$

where the subscripts $l$ and $v$ represent the liquid and vapor phases, respectively.

The equilibrium co-existence state of the system can be derived by minimizing the free-energy functional $F$ under the constraint of mass conservation, which leads to a second-order stress tensor in the form of (for the detailed derivation, the interested reader is referred to[47,58,59])

$$
\vec{\Pi}=\left[p(\rho, T)-\kappa \rho \nabla^{2} \rho-\frac{1}{2} \kappa|\nabla \rho|^{2}\right] \vec{I}+\kappa \nabla \rho \otimes \nabla \rho .
$$

Let $\vec{I}$ denote the identity tensor, and the thermodynamic pressure for a van der Waals fluid is given by

$$
p(\rho, T)=\rho \frac{\partial f(\rho, T)}{\partial \rho}-f(\rho, T)=\frac{k_{B} T N_{V}(\rho)}{1-b N_{V}(\rho)}-a N_{V}(\rho)^{2} .
$$

With the divergence-free extended pressure tensor Eq. (4), we finally arrive at the nonequilibrium model for a viscous compressible two-phase flow with a finite-width interface:

$$
\begin{gathered}
\frac{\partial \rho}{\partial t}+\nabla \cdot(\rho \vec{v})=0 \\
\frac{\partial}{\partial t}(\rho \vec{v})+\nabla \cdot(\rho \vec{v} \otimes \vec{v})=\nabla \cdot \vec{\tau}-\nabla \cdot \vec{\Pi}+\rho g \vec{e}_{g},
\end{gathered}
$$


where $\vec{v}$ is the fluid velocity, $\vec{e}_{g}$ is the unit vector in the direction of the gravitational force, and $g$ is the acceleration due to gravity. Based on the Stokes' hypothesis, the viscous stress tensor is given as $\vec{\tau}=\eta\left\{\left[\nabla \vec{v}+(\nabla \vec{v})^{T}\right]-2 \vec{I} \nabla \cdot \vec{v} / 3\right\}$, with $\eta$ denoting the shear viscosity.

For a non-isothermal system such as boiling, the governing equations (6) and (7) need to be appended by the balance equation for energy. It should be mentioned that the local energy flux ought to include both classic (based on Fourier's law of heat conduction) and nonclassic (due to interstitial working[47]) contributions, which leads to

$$
\frac{\partial e}{\partial t}+\nabla \cdot(e \vec{v})=(\vec{\tau}-\vec{\Pi}): \nabla \vec{v}+\nabla \cdot\left(\lambda \nabla T-\kappa \frac{D \rho}{D t} \nabla \rho\right)
$$

In accordance with the van-del-Waals equation of state, the internal energy here can be evaluated as $e(\varrho, T)=f(\varrho, T)-T \partial f(\varrho, T) / \partial T=3 k_{B} T N_{V}(\varrho) / 2-a N_{V}(\varrho)^{2}$. Furthermore, we postulate that simple linear dependences on density exist for the viscosity $\eta$ and the thermal conductivity $\lambda$, which essentially entails[47,48,59]

$$
\begin{aligned}
& \eta=\eta_{0} \rho, \\
& \lambda=\lambda_{0} \rho .
\end{aligned}
$$

It is worth noting that alternatively, instead of the energy flux, the pressure tensor itself (Eq. (4)) can be modified to account for the effect of adiabatic interfacial forcing[61]. It appears that these two formulations will lead to more or less indistinguishable predictions for heterogeneous boiling behavior under constanttemperature boundary heating[59].

\section{B. Nondimensionalization}

The equations (6), (7), and (8), along with the equation of state (Eq. (5)) constitute the set of governing partial differential equations, which can be solved numerically in nondimensional form. The critical coordinates of water $\left(T_{c}=8 a / 27 k_{B} b=647.1 \mathrm{~K}, p_{c}=a / 27 b^{2}=22.1 \mathrm{MPa}\right.$, and $\left.\varrho_{c}=m_{p} / 3 b=322.0 \mathrm{~kg} / \mathrm{m}^{3}\right)$ are used 
as the scales for temperature, pressure, and density, respectively. Specifically, the reduced fluid variables read

$$
\begin{gathered}
\tilde{T}=T / T_{c}, \\
\tilde{p}=p / p_{c}, \\
\tilde{\rho}=\rho / \rho_{c}, \\
\tilde{e}=e \frac{27 b^{2}}{a}=e / p_{c},
\end{gathered}
$$

where the tildes mean dimensionless quantities.

In order to fully resolve the diffuse interface, the length-scale is chosen to be $l^{*}=2 b^{1 / 3}=7.4 \times 10^{-10} \mathrm{~m}, \quad$ and the matching time-scale hence becomes $t^{*}=6 b^{5 / 6}\left(m_{p} / a\right)^{1 / 2}=2.8 \times 10^{-12} \mathrm{~s}$. Such miniscule values pose one of the main challenges facing the phase-field-based approach-rendering it extremely difficult, if not impossible, to perform meaningful quantitative comparisons with experiments except for cases involving critical phenomena or nanofluidic applications. However, we deem that the benefits of using the diffuse-interface method outweighs the disadvantages for it can lead to valuable and, more importantly, theoretically sound insights regarding the interfacial interactions[47].

Based on the thermophysical properties of saturated water at $T=0.5 T_{c}$, we adopt the following dimensionless parameters: $\tilde{\eta}_{0}=1.0, \tilde{\lambda}_{0}=30.0$, and $\tilde{\kappa}=1.0$. More details with respect to the nondimensionalization can be found in[59]. Special care is taken when handling the external-force term in the momentum equation (7), which involves the nondimensional gravitational acceleration in the form of

$$
\tilde{g}=\frac{t^{* 2}}{l^{*}} g
$$

Given that $t^{*}$ and $l^{*}$ are both negligibly small, instead of the terrestrial value of 9.8 $\mathrm{m} / \mathrm{s}^{2}$, we rely on an artificial gravity, which incidentally needs to be inflated multiple orders of magnitude[48,62], to impose any meaningful impacts on bubble growth and, for that matter, contact-line propagation. (Actually, the effect of the consequently different bubble growth rates on contact-line motion will constitute the main focus of 
the following sections.) For the sake of conciseness and readability, without specific indication, the accents ${ }^{\sim}$ will be dropped henceforth from the dimensionless variables.

\section{Numerical scheme}

We employ the finite-element-based symbolic computing toolbox FemLego[63], which features the characteristic-based split (CBS) scheme, to numerically solve the partial differential equations. Temporal terms are discretized using the first-order Euler forward method, whereas space discretization relies on the piecewise linear approximation with adaptive mesh refining in the vicinity of the liquid-vapor interface. The general minimal residual (GMRES) algorithm is used to solve the resulting linear systems with an iterative convergence tolerance set at a relatively strict threshold of $10^{-8}$. The interested reader is referred to[59] for more technical details about the computational procedures and code verification.

Figure 1(a) shows the two-dimensional asymmetrical computation domain $(0<r \leq 200$ and $0 \leq z \leq 300)$ used in the present study, which is filled with saturated liquid water at $T_{\text {sat }}=0.79$ and $\varrho_{\text {sat }, l}=1.95$. Note that in order to avoid computational uncertainties associated with the cylindrical-coordinate singularity, the axis of symmetry is purposefully removed from the left boundary (located at $r=0.001$ ), where the symmetry boundary condition is enforced.

The open boundary condition[59], which includes

$$
\begin{gathered}
\nabla_{n} \vec{v}=0 ; \\
\rho=\rho_{s a t, l} ; \\
T=T_{s a t} ; \\
\prod_{i j}=p_{s a t}\left(T_{s a t}\right) \delta_{i j},
\end{gathered}
$$

is assigned to the top boundary $(z=300)$. Here $\delta_{i j}$ denotes the Kronecker delta, and $\nabla_{n}$

the gradient normal to the boundary. Essentially connecting the domain with an infinite virtual reservoir of saturated liquid, the open boundary condition approximates vigorous boiling under the external pressure of $p_{\text {sat }}=0.29$. Such a treatment is 
equivalent to maintaining a metastable liquid phase by placing a gaseous pocket above the growing bubble[62].

At the bottom $(z=0)$ and right walls $(r=200)$, the no-slip condition can be safely imposed since the singularity of infinite stress at a moving contact line is readily resolved under the diffuse-interface framework[56]. Following the localized surfaceenergy approach[59], we model the wetting boundary condition at an isothermal solid wall as

$$
\nabla_{n} \rho=\frac{\sigma \cos \theta_{s}}{\kappa} \partial_{\rho} \psi(\rho)
$$

Such a relation is derived from applying energy minimization again at the contact line, which leads to fast relaxation of the density distribution that approximates the equilibrium microscopic contact angle $\theta_{s}$. Note that $\Psi(\varrho)$ represents an interpolating third-order polynomial function of density that satisfies $\Psi\left(\varrho_{s a t, v}\right)=0$ and $\Psi\left(\varrho_{s a t, l}\right)=1$, along with the constraints $\partial_{\varrho} \Psi\left(\varrho_{s a t, v}\right)=0$ and $\partial_{\varrho} \Psi\left(\varrho_{s a t, l}\right)=0$ (such that Eq. (20) is nonzero only in the interfacial region).

It should be mentioned that the wetting condition can be described alternatively by a so-called geometric formulation, which was claimed to result in more faithful reproduction of the imposed contact angle in, for instance, droplet spreading[64]. Notwithstanding, as was shown in one of our previous studies[59], the evolution of the particular interfacial shape under the surface-energy formulation eventually arrives at an apparent contact angle that closely matches (within 3 degrees) the set angle $\theta_{s}$. Hence, we continue to use Eq. (20) in the present study. While the right wall has a trivial value of $\theta_{s}=90^{\circ}$, the bottom wall is divided into a hydrophobic region $(r \leq 60.0)$ featured by $\theta_{s, p h o}$ and a hydrophilic region $(r>60.0)$ by $\theta_{s, p h i}$, which notably constitutes a biphilic surface.

To simulate the robust growth of a vapor bubble in pool boiling, at $t=0$ a spherical bubble nucleus $\left(\varrho_{v}=0.31, T_{v}=0.73\right.$, and $\left.p_{v}=0.38\right)$ with an initial radius of 50.0 is artificially seeded atop the bottom wall, whose center is located at $z=10.0$. The interfacial density profile follows a hyperbolic tangent function set between $\varrho_{v}$ and $\varrho_{l}[60]$ over a tentative width of 5.0. Additionally, an isothermal (Dirichlet-type) 
condition is imposed at the bottom wall (namely, at a superheat of $\Delta T=0.06$ ). The computational domain is discretized by a $200 \times 300$ mesh. All simulation runs are carried out under a uniform time step of $\Delta t=0.01$.

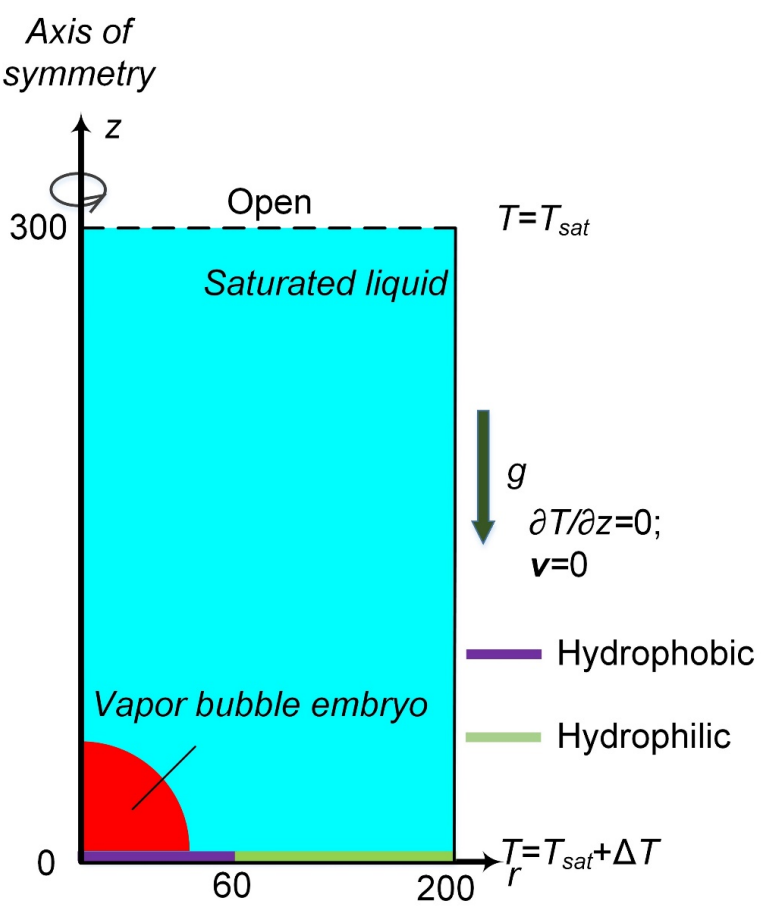

Fig. 1. Schematic illustration of the axisymmetric simulation domain. The top open boundary, which allows free flow of saturated liquid, mimics saturated heterogeneous boiling under surface superheating of $\Delta T$. The initiation of boiling is realized by seeding a vapor bubble nucleus on the top of the heating wall. By use of the surface-energy formulation, contrasting wetting conditions can be applied to the bottom boundary, with the hydrophobic (marked in purple) and hydrophilic (marked in green) regions demarcated at $r=60.0$.

\section{CONTACT-LINE DYNAMICS}

In this section, we will study in many details the contact-line dynamics traversing a wettability divide on the solid surface under various gravities. It is worth noting that in choosing gravity as the sole parameter of interest, we can effectively eliminate other interfering factors such as various thermophysical properties. Moreover, because of the strong correlation between the gravity level and the bubble expansion rate in boiling[65], the results are expected to shed light on the unalloyed relationship of the bubble growth rate versus the pinning state of contact line as it straddles the border between contrasting hydrophobic and hydrophilic surfaces. 


\section{A. Low gravity $(g=0.0002)$}

We plot in Fig. 2 the time evolution of bubble shape (namely, the density distribution) as it is growing on the biphilic surface with $\theta_{s, p h o}=130^{\circ}$ and $\theta_{s, p h i}=10^{\circ}$, under a low gravity level of $g=0.0002$. Due to the strong hydrophobicity of the surface underneath the bubble, the initial fast expansion of the contact line far precedes that of the rest of the bubble, as is evidenced by the protruding bubble base at $t=0.5$. Once the bubble grows beyond the border of the hydrophobic surface, however, the contact line appears to drag behind while the bubble continues growing, which results in a shift in the apparent contact angle that more or less reflects the surface's nominally enhanced affinity for water. 

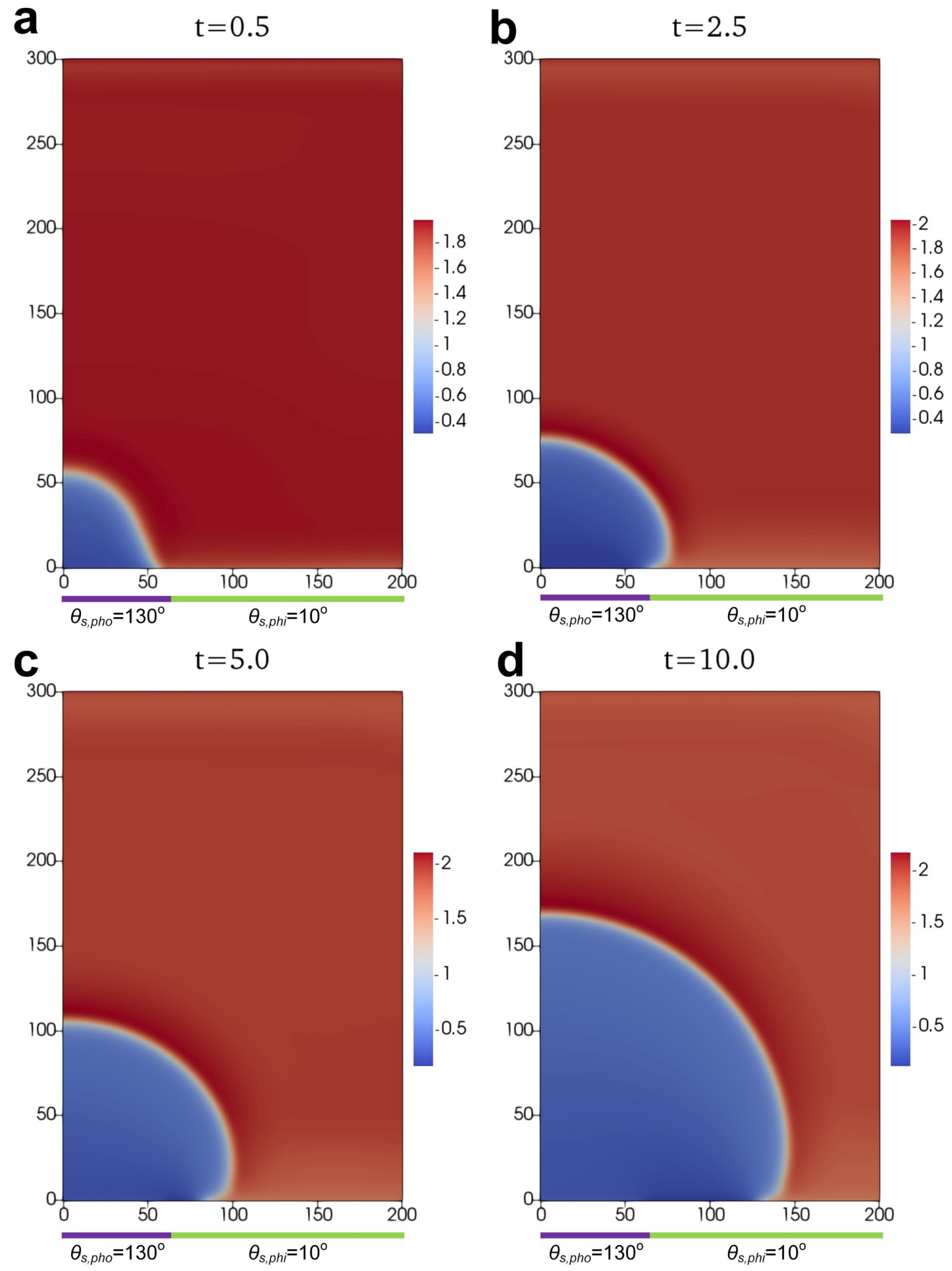

Fig. 2. Snapshots of the density distribution at different times for the case with $g=0.0002$ at (a) $t=0.5$, (b) $t=2.5$, (c) $t=5.0$, and (d) $t=10.0$. The initial stage of the bubble growth features fast expansion of the bubble base on the hydrophobic surface (marked in purple, with $\theta_{s, p h o}=130^{\circ}$ ).

The local convex bubble outline near the heating surface quickly turns into a concave shape once 
the contact line enters the hydrophilic territory (marked in green) at $r=60.0$, due largely to a shift in the surface wetting characteristics $\left(\theta_{s, p h o}=10^{\circ}\right)$.

A more quantitative depiction of the (horizontal) bubble growth on the biphilic surface can be found in Fig. 3, which traces the reaches of the bubble-base (contactline radius $R_{c}$ ) and the bubble itself (bubble width $R_{b}$, as defined using the rightmost point along the bubble interface) over time. Because the contact-line expansion leads the bubble growth during the initial bubble spreading on the hydrophobic surface, the results for $R_{c}$ and $R_{b}$ overlap with each other until around $t=1.2$. Then, a bifurcation starts to emerge between $R_{c}$ and $R_{b}$ when the bubble reaches the end of the hydrophobic region and the contact line gets overtaken by the rest of the bubble. Specifically, the contact-line motion faces strong resistance as it strives to overcome the wettability divide, as shown by the sharp decline of the corresponding timederivative $\dot{R}_{c}$, whereas the bubble itself keeps growing. Note that the marked hinderance to the contact-line expansion appears to be only temporary, since the pace of the contact-line expansion quickly recovers and catches up to that of the remaining bubble after the contact line finally moves over onto the hydrophilic part of the surface. 


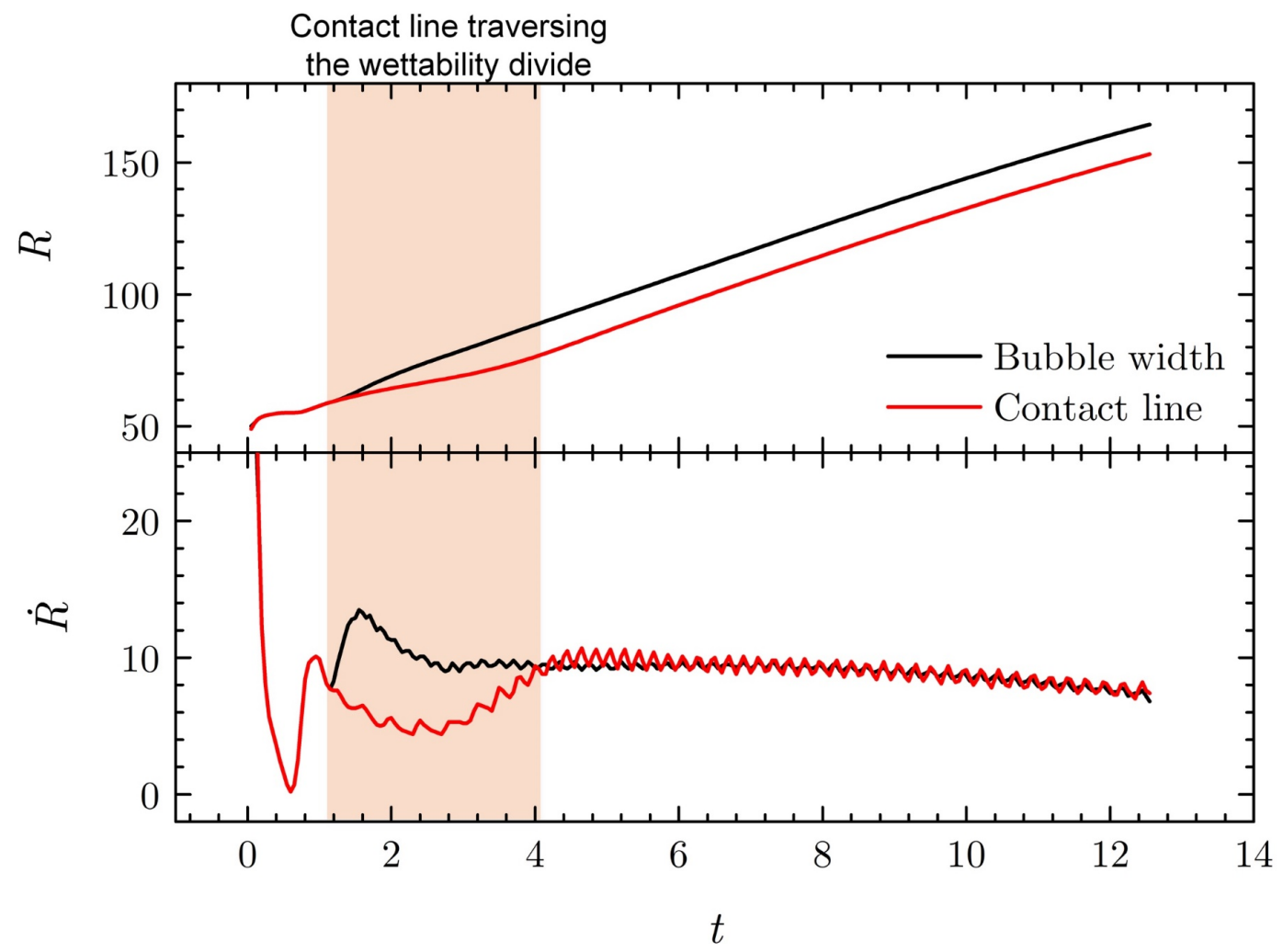

Fig. 3. Plots of the bubble width $R_{b}$ (in black) and the radius of the contact line $R_{c}$ (in red) as functions of time and their corresponding time derivatives $\dot{R}_{b}$ and $\dot{R}_{c}$, for the case with $\theta_{s, p h o}=130^{\circ}, \theta_{s, p h i}=10^{\circ}$, and $g=0.0002$. The expansion of the bubble contact line seems to encounter a pronounced yet brief interruption as it traverses the border between the hydrophobic and the hydrophilic surfaces.

\section{B. High gravity $(g=0.0007)$}

Next we repeat the simulation but with an elevated gravity $g=0.0007$ while keeping everything else unchanged. The results describing bubble growth are shown in Fig. 4. Quite similar to the case with the low gravity (cf. Fig. 2), the bottom of the bubble seems to be expanding at an increasing rate on the hydrophobic surface at the early time of $t=0.5$, which leads to a bell-like shape. But what transpires after the contact line arrives at the interface of the contrasting wettabilities differs dramatically from the previous case. Instead of being only temporarily delayed, the contact line appears to remain pinned at the boundary (around $r=60.0$ ) between the hydrophobic and hydrophilic regions. Thereafter, the abruptly interrupted horizontal expansion 
leaves bubble to grow mainly along the vertical direction. It is interesting to note that on account of the continuous pinning of the contact line, the bubble interface near the wall appears to turn increasingly stretched as the bubble grows, giving rise to formation of a sharp contact angle extending all the way to the midsection of the bubble at $t=25.0$. More discussion regarding the apparent contact angle will follow in Sec. IV. 

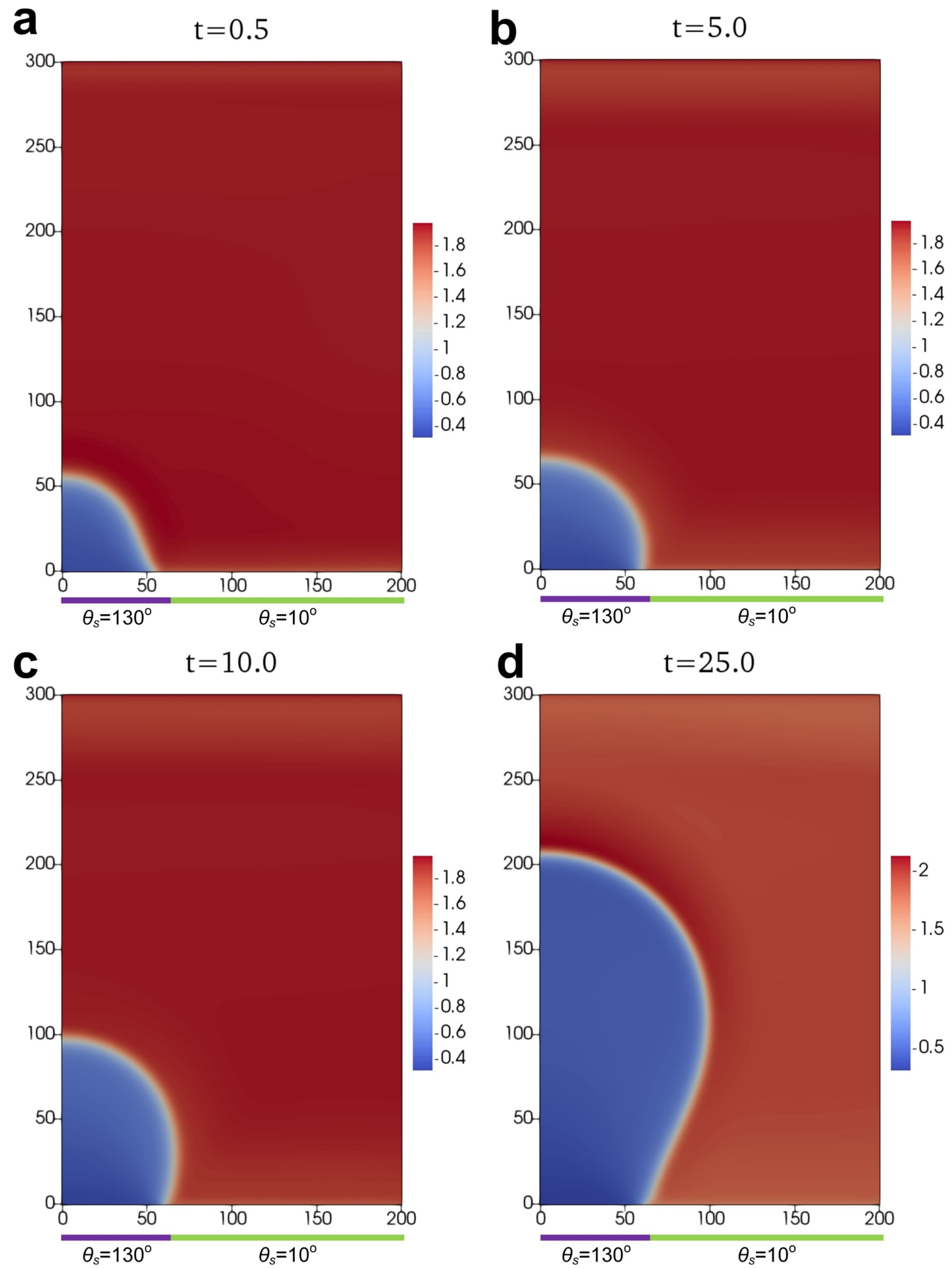

Fig. 4. Snapshots of the density distribution for the case with $g=0.0007$ on a biphilic surface divided at $r=60.0$ between the hydrophobic section (marked in purple, $\theta_{s, p h o}=130^{\circ}$ ) and the hydrophilic section (marked in green, $\theta_{s, p h o}=10^{\circ}$ ) at (a) $t=0.5$, (b) $t=5.0$, (c) $t=10.0$, and (d) $t=25.0$. The bubble contact line is seen to get pinned at the wettability divide while the bubble 
continues to grow vertically, which gives rise to the continued stretching of the lower half of the bubble interface all the way down to the surface.

Similarly, we show plots of $R_{b}$ and $R_{c}$ as functions of time and their timederivatives in Fig. 5. Compared with their low-gravity counterparts in Fig. 3, here the bubble appears to grow appreciably slower. We notice that under such significantly decelerated bubble growth, the contact-line propagation on the surface eventually comes to a halt $\left(\dot{R}_{c} \approx 0\right)$ at the entrance to the hydrophilic region around $r=60.0$, while the rest of the bubble continues to grow (even at an apparently accelerated rate), in sharp contrast to Fig. 3. In the inset we show enlarged results depicting a gradual drastic slowdown, rather than a complete termination, of the contact-line expansion, which agrees with recent molecular dynamics (MD) simulations of droplet evaporation under the effect of surface heterogeneities $[42,66]$. Moreover, the pinning of the contact line at the wettability border seems to be particularly strong since no re-convergence occurs between the curves for $\dot{R}_{b}$ and $\dot{R}_{c}$ for the time period considered. In the case with $g=0.0002$ (see Fig. 3), by contrast, the depinned contact line finds itself being dragged along by the continuous bubble expansion on the hydrophilic surface, which results in $\dot{R}_{c}$ moving in almost perfect tandem with $\dot{R}_{b}$. Also noted is the apparently elevated level of "noises" present in the results of $\dot{R}_{b}$ and $\dot{R}_{c}$ in Fig. 3, in comparison with Fig. 5. One possible explanation is that the increased instability could be caused by a propagating capillary wave along the bubble interface that is generated from the moving contact line[67]. 


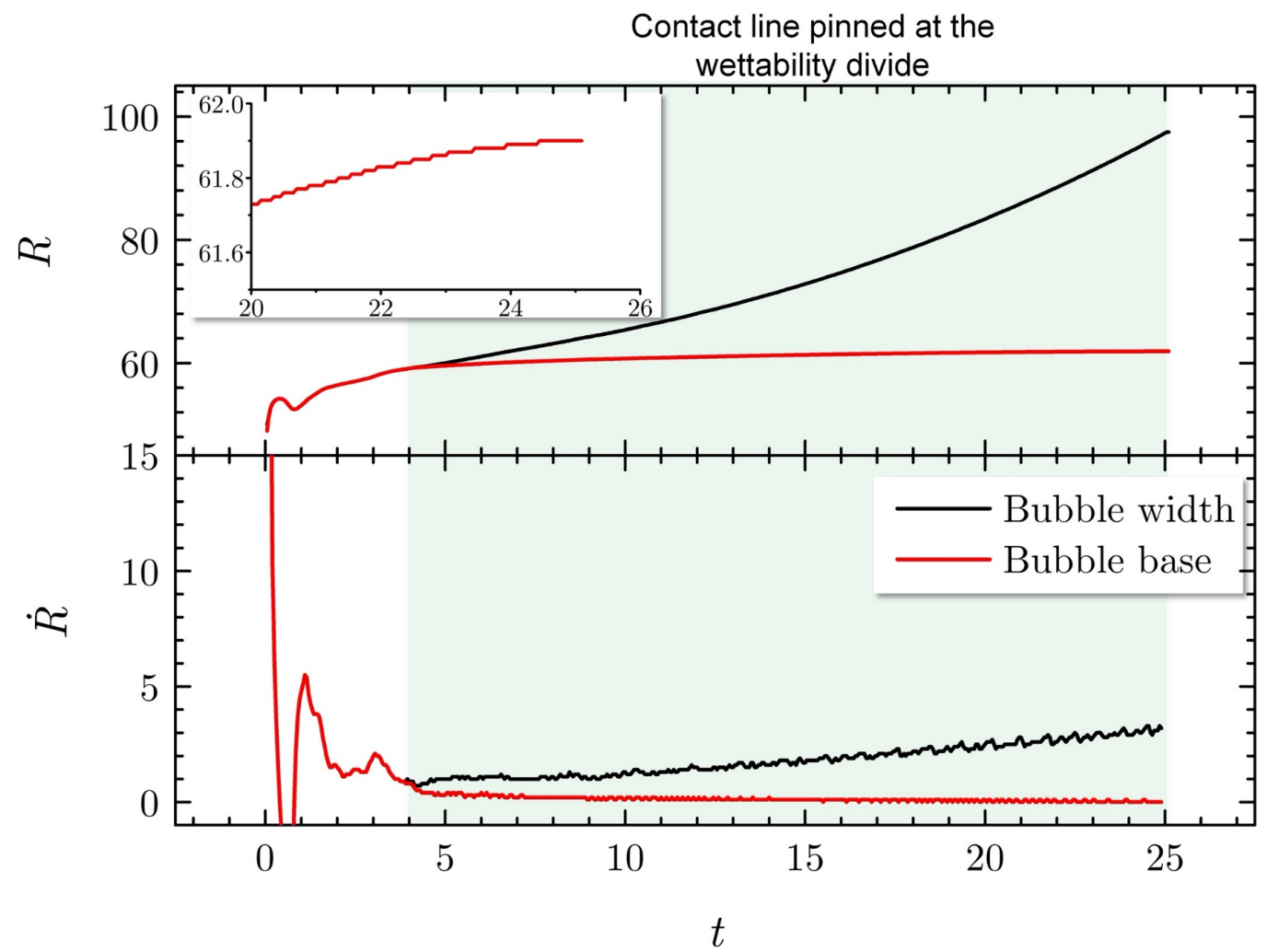

Fig. 5. Plots of the bubble width $R_{b}$ (in black) and the radius of the contact line $R_{c}$ (in red) as functions of time and their corresponding time derivatives $\dot{R}_{b}$ and $\dot{R}_{c}$, for the case with $\theta_{s, p h o}=130^{\circ}, \theta_{s, p h i}=10^{\circ}$, and $g=0.0007$. The results show clearly that the contact line fails to travel beyond the hydrophobic surface but remains hemmed in by the surrounding hydrophilic surface $(r=60.0)$. Inset: enlarged view showing long-term slowing down of the contact-line expansion.

\section{From depinning to pinning}

As the above comparison of the cases of low and high gravities has shown, the level of gravity has interesting implications for contact-line behavior on a mixedwettability surface. Having performed more simulations under the same surface wettability contrast $\left(\theta_{s, p h o}=130^{\circ}\right.$ and $\left.\theta_{s, p h i}=10^{\circ}\right)$ but at different $g$ 's, we plot in Fig. 6 with growing gravity the variations of the characteristic traversing bubble expansion rate $\left\langle\dot{R}_{b}\right\rangle$ and the corresponding contact line speed $\left\langle\dot{R}_{c}\right\rangle$, respectively. The results represent the transition from the depinned-contact-line (DCL) mode to the pinnedcontact-line (PCL) mode. Note that here $\left\langle\dot{R}_{b}\right\rangle$ and $\left\langle\dot{R}_{c}\right\rangle$ are calculated averages based on the extracted data of $\dot{R}_{b}$ and $\dot{R}_{c}$ over the duration of wettability-border 
crossing when the contact line dynamics is evidently under the influence of the wettability contrast (for instance, the highlighted parts in Figs. 3 and 5). The error bars denote data spread (as defined by standard deviation of the data set).

It seems that the variation of $\left\langle\dot{R}_{c}\right\rangle$ is nearly parallel to that of $\left\langle\dot{R}_{b}\right\rangle$, which attests to the strong correlation between the contact-line dynamics and the bubble growth rate in heterogeneous boiling. As $g$ increases, both $\left\langle\dot{R}_{c}\right\rangle$ and $\left\langle\dot{R}_{b}\right\rangle$ diminish following quasi-linear relations. Around $g=0.0005$, a noticeable departure from the steep-sloped pattern begins to emerge, indicating a fundamental shift in the behavior of bubble spreading on the surface. With further increases in $g$ the falling general bubble growth rate gradually levels off, whereas the contact line speed is reduced to vanishingly small values. Hence, such a dramatic transition suggests that a complete demobilization of contact line could indeed result from considerable deceleration of bubble expansion across the surface-wettability gap. In what follows, we will delve further into the different contact-line characteristics between the DCL and PCL modes and their transition under the influence of surface wettabilities. 


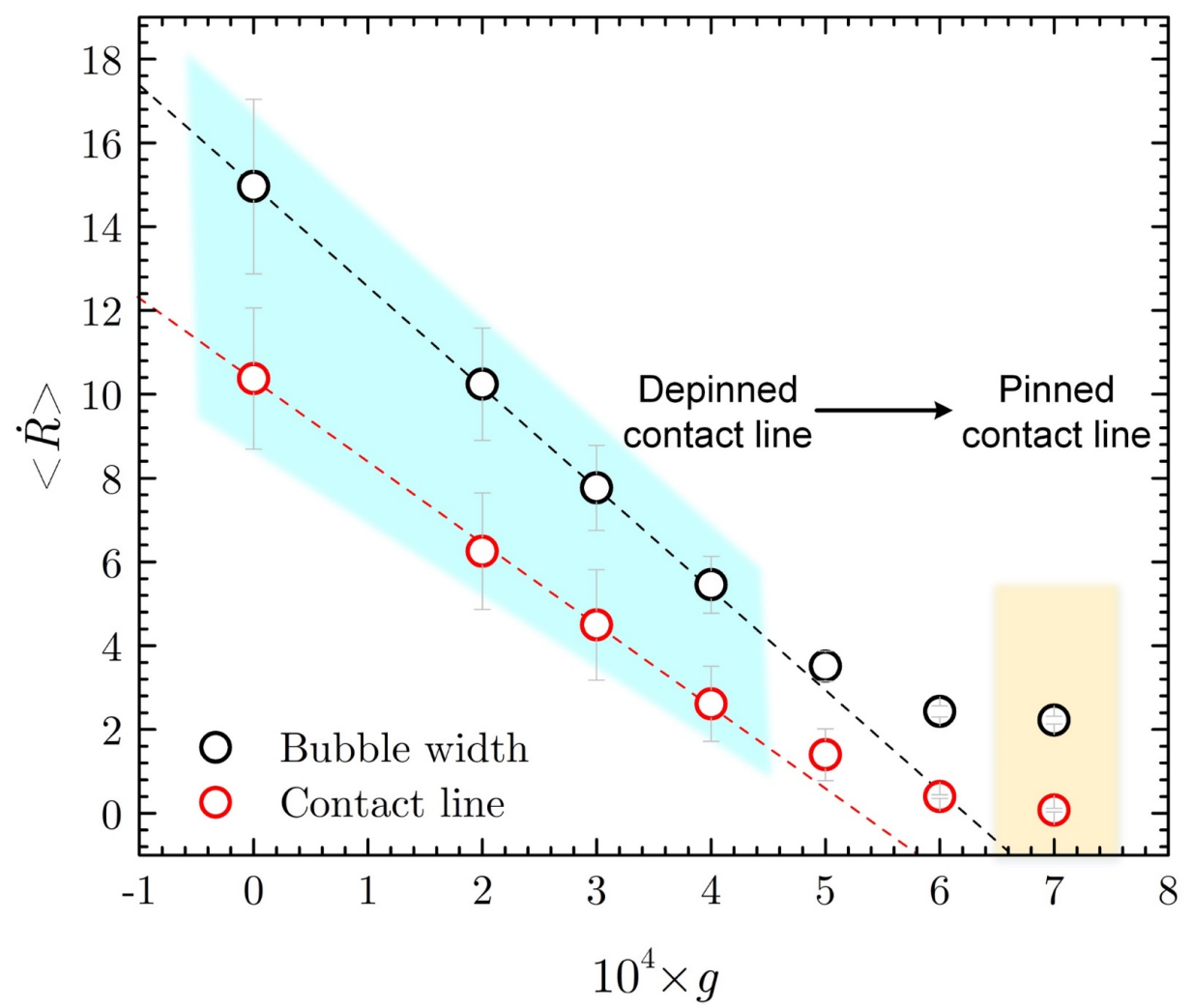

Fig. 6. Variations of the average bubble growth rate $\left\langle\dot{R}_{b}\right\rangle$ (black circles) and contact-line speed $\left\langle\dot{R}_{c}>\right.$ (red circles) during the contact-line crossing at the wettability divide $\left(\theta_{s, \text { pho }}=130^{\circ}\right.$ vs. $\left.\theta_{s, p h i}=10^{\circ}\right)$ on the surface over different gravities. Completely depinned contact lines appear to dominate under the effect of low gravity, which, in response to the slowing-down of bubble expansion, becomes less mobile following a similar linear relationship with $g$ (marked by dash lines). When the gravity level is sufficiently high, however, $\left\langle\dot{R}_{c}\right\rangle$ eventually drops to nearly zero, which indicates pinning of the contact line at the wetting border. The error bars represent standard deviations of the data sets.

\section{EFFECT OF HETEROGENEOUS WETTABILITIES}

Encouraged by the promising results depicting divergent contact-line dynamics under the effect of the bubble expansion rate, we now set out to broaden the scope of the study to elucidate the effect of the surface wetting pattern. The objective is to derive a more complete description of the behavior of contact line traversing wetting heterogeneities. In what follows, a series of simulations are performed based on the 
same problem setup but with different combinations of $\theta_{s, p h o}$ and $\theta_{s, p h i}$. The results reveal some remarkable characteristics of the contact-line mobility in the DCL mode, and the interesting emergence of seemingly universal (effective) contact angle in the PCL mode.

\section{A. Contact-line mobility}

In Fig. 7 we show results delineating the influence of the biphilic pattern (that is, the contrast between $\theta_{s, p h o}$ and $\left.\theta_{s, p h i}\right)$ on the dynamics of a more mobile contact line (namely, under the DCL regime) crossing the wettability interface. Here the vertical axes denote the relative movability of the traversing contact line, which is defined as

$$
\Gamma=\frac{\left\langle\dot{R}_{c}\right\rangle}{\left\langle\dot{R}_{b}\right\rangle} .
$$

Equation (21) measures the extent to which the contact line spreading gets slowed down as it is pulled by the expanding bubble across from the hydrophobic section onto the hydrophilic section of the surface. 
a

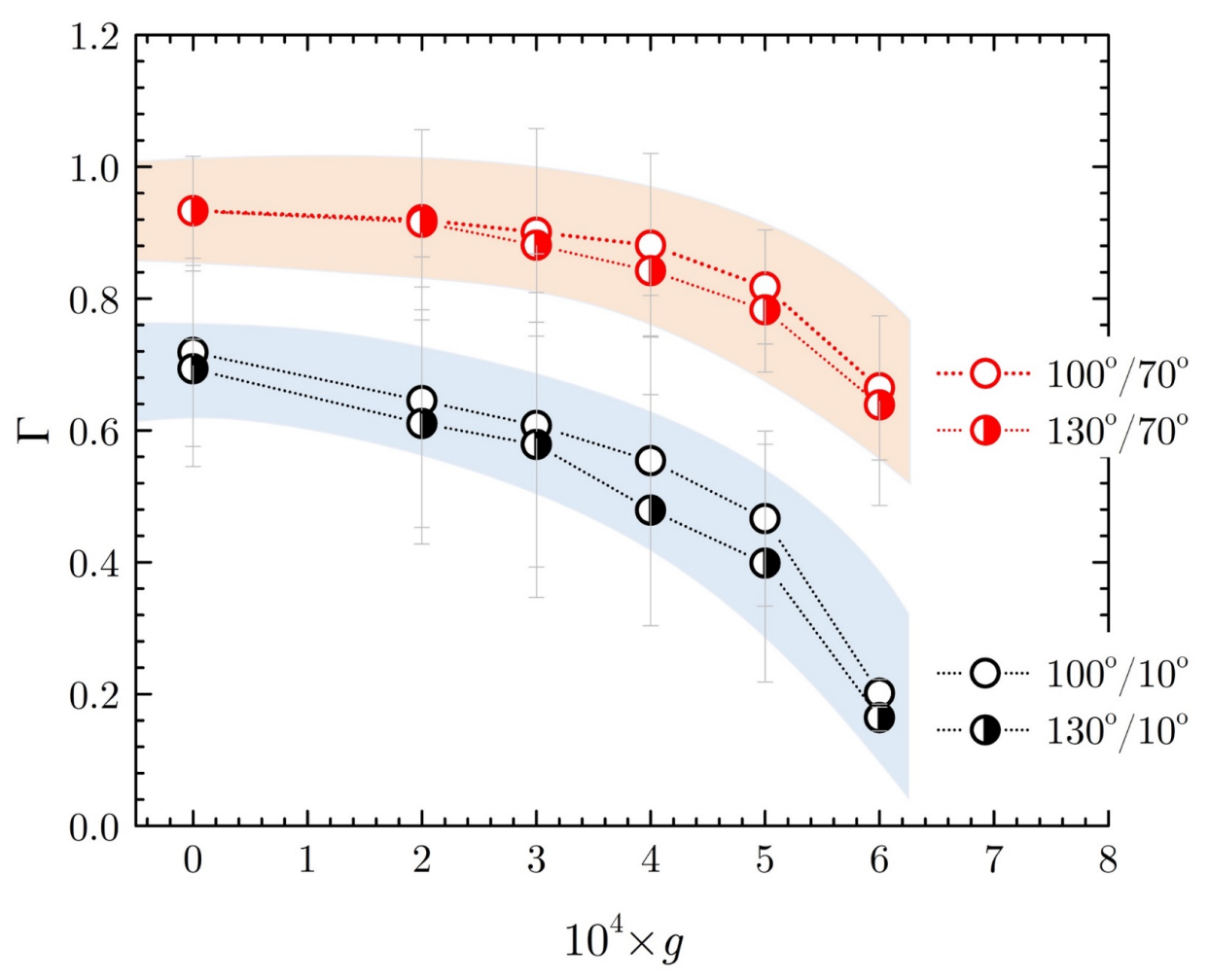

b

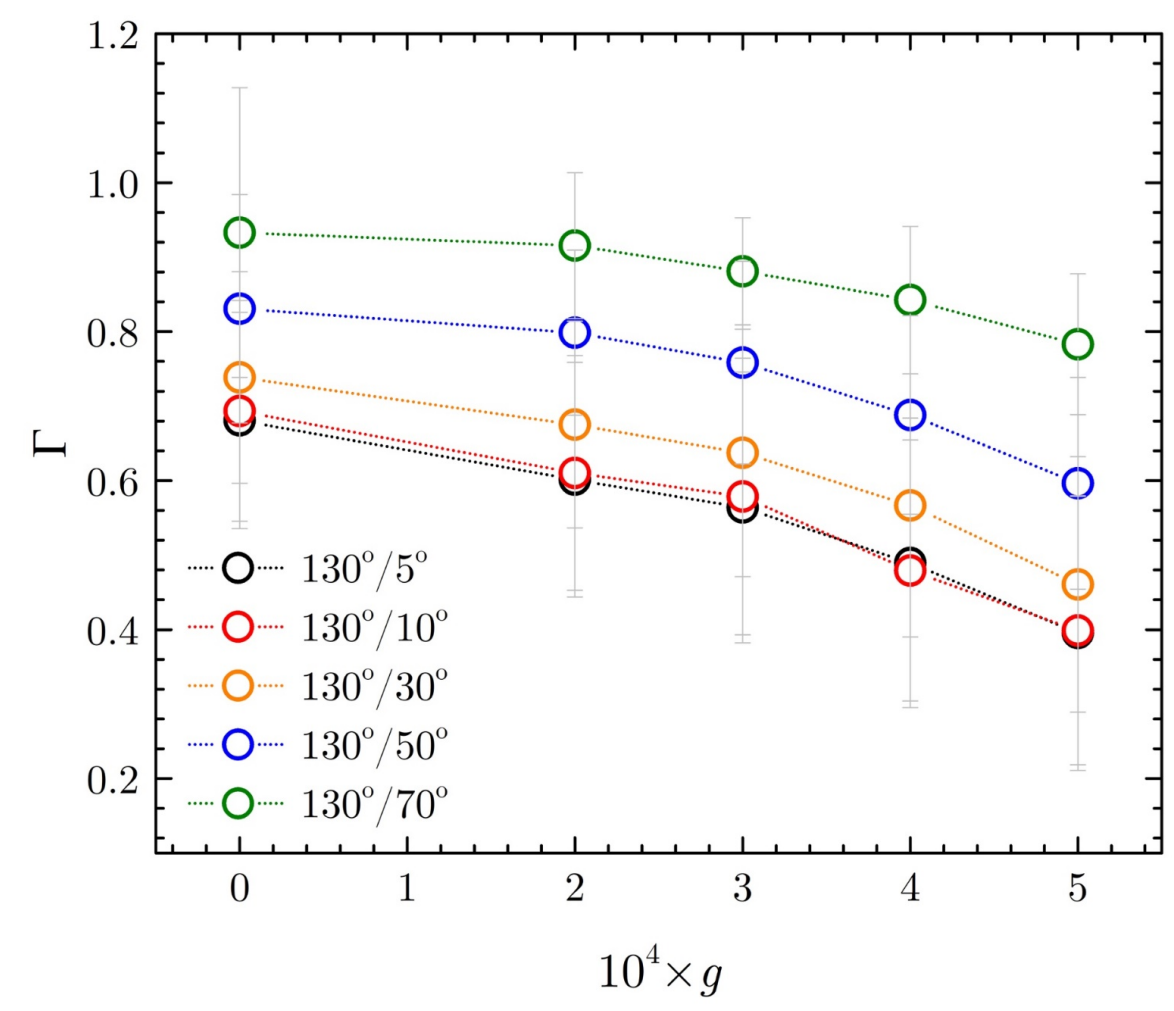


Fig. 7. Contact-line mobility as defined by the ratio of the traversing contact-line speed to the bubble expansion rate, $\Gamma=\left\langle\dot{R}_{c} / \dot{R}_{b}\right\rangle$, under the influence of the wettability contrast of $\theta_{s, \text { pho }}$ and $\theta_{s, p h i} . \ln (\mathrm{a})$, the results demonstrate a quite moderate effect of the imposed contact angle $\theta_{s, \text { pho }}$ of the hydrophobic surface $(r \leq 60.0)$ on the overall distribution of $\Gamma$ at different gravities. In $(b)$, on the other hand, $\Gamma$ is found to be more sensitive to the changing affinity for water (measured by $\left.\theta_{s, p h i}\right)$ of the hydrophilic part of the surface $(r>60.0)$. Note that the error bars denote the datareduction uncertainties.

First, we examine the effect of surface hydrophobicity as is defined by $\theta_{s, p h o}$. As Fig. 7 (a) shows, $\Gamma$ falls sharply when the gravity level is raised from zero to $g=0.0006$ : from an initial level close to $\Gamma=0.9$ down to around $\Gamma=0.7$ in the cases (marked in red) of $\theta_{s, p h o}=100^{\circ}\left(\right.$ with $\left.\theta_{s, p h i}=70^{\circ}\right)$ and $\theta_{s, p h o}=130^{\circ}\left(\right.$ with $\theta_{s, p h i}=70^{\circ}$ ), and from $\Gamma=0.7$ down to a paltry $\Gamma=0.2$ in the cases (marked in black) of $\theta_{s, p h o}=100^{\circ}$ (with $\theta_{s, p h i}=10^{\circ}$ ) and $\theta_{s, p h o}=130^{\circ}$ (with $\theta_{s, p h i}=10^{\circ}$ ). The consistently diminishing contact-line mobility relative to the general bubble expansion rate indicates growing resistance in the presence of clear wettability contrast, which, incidentally, will lead the contact line to an ultimate standstill if $g$ is allowed to increase even further (see Fig. 6). More interestingly though, the variations of $\Gamma$ appear to depend only weakly on the assigned hydrophobic contact angle since the results differ ostensibly less between the cases with different $\theta_{s, p h o}$ 's than those with different $\theta_{s, p h i}$ 's. In other words, the dynamics of propagating contact lines across opposing wettabilities seems to rely mainly on the more wettable side of the divide.

Such a dominant role of surface hydrophilicity is made clear in Fig. 7(b), which plots similar calculations of $\Gamma$ for varying degrees of water affinity (of the hydrophilic section, $r>60.0$ ) ranging from $\theta_{s, p h i}=5^{\circ}$ up to $\theta_{s, p h i}=70^{\circ}$, while the assigned equilibrium hydrophobic contact angle is maintained at a constant value of $\theta_{s, p h o}=130^{\circ}$. Besides the similar trend of steadily dwindling $\Gamma$ with increasing $g$, one can detect increasing retardation to the contact-line motion on the more hydrophilic surface for $\Gamma$ decreases ever lower when $\theta_{s, p h i}$ lessens. The apparently strong dependence of $\Gamma$ on $\theta_{s, p h i}$ can be explained in a somewhat straightforward manner. On account of the specific direction 
of contact-line propagation (i.e. from the hydrophobic part to the hydrophilic part of the surface in the present simulation), it is reasonable to expect the motion to be affected more by the hydrophilic surface ahead. Furthermore, it is also more likely to encounter greater resistance in displacing liquid with vapor, as does the moving contact line, on the surface that exhibits apparent preference for the former.

It is noteworthy that the impedance to the traversing contact line across the wettability divide seems to 'saturate' at $\theta_{s, p h i}=10^{\circ}$, beyond which point further decreases in $\theta_{s, p h i}$ only brings about marginal reductions to the contact-line mobility. This raises the implication of diminished effectiveness of enforcing contact-line pinning, in order to avoid the transition to low-efficiency intermittent boiling on biphilic surfaces, by merely enlarging the wettability gap alone.

\section{B. Apparent contact angle}

Despite the imposed wetting boundary conditions through the equilibrium contact angle $\theta_{s, p h o}$ and $\theta_{s, p h i}$, the liquid-vapor interface finds itself meeting the solid surface at a somewhat different apparent angle, which is susceptible to (local) distortions due to dynamic effects in the contact-line region[59]. Figure 8 describes the typical effective contact angles $\theta_{a}$ that forms at the depinned and pinned contact lines on the biphilic surface (with $\theta_{s, p h o}=130^{\circ}$ and $\theta_{s, p h i}=10^{\circ}$ ), respectively. One should note that $\theta_{a}$ in the present study differs from the conventionally defined dynamic contact angle[64]. In both hydrodynamic[68] and molecular kinetic interpretations[55], the dynamic contact angle is defined through a constitutive relation dominated by the speed of contact-line motion, which in turn relies on a variety of factors including surface roughness and chemical inhomogeneities. In the present simulation, as is shown below, the resulting apparent contact angles exhibit no clear dependence on the velocity of the contact line (or the bubble growth rate at different $g$ 's).

Figure $8(\mathrm{a})$ shows that, in the DCL case $(g=0.0002)$, subsequent to it overcoming the energy barrier due to the wettability contrast, the contact line goes on to freely spread on the hydrophilic surface, closely following the rest of the bubble (see Fig. 3). The local equilibrium in the contact-line region under the imposed wetting 
condition of $\theta_{s, p h i}=10^{\circ}$ results in sharp bending of the interfacial profile very close to the surface. Consequently, the effective contact angle $\theta_{a}$ turns out to be mostly confined to a thin layer between the solid surface and the rapidly expanding bubble. Many details about the contact-line structure are hence hidden beneath the smooth interface of the macroscopic bubble.

By contrast, in the PCL case $(g=0.0007)$, the contact line remains firmly anchored upon reaching the edge of the hydrophilic surface (around $r=60.0$ ). Too weak to dislodge the contact line, the bubble growth thereafter proceeds along the stretched bubble interface at an inclined (contact) angle to the solid surface, $\theta_{a}$, as shown by Fig. $8(\mathrm{a})$. Note that thanks to the strong pinning of the contact line, $\theta_{a}$ prevails beyond the immediate vicinity of the surface and over the entire lower half of the bubble (see Fig. 4(d)).

According to the evolution history of $\theta_{a}$, which is shown in Fig. 8(b), the apparent contact angle in the PCL mode quickly settles into a constant value once the contact line gets pinned on the surface. The continuous bubble expansion appears to have little if any effect on $\theta_{a}$. For the DCL modes, on the other hand, the results show that $\theta_{a}$ declines precipitously as the contact line ventures onto the hydrophilic surface after a brief delay. Moreover, compared with the relatively smooth $\theta_{a}$ on the hydrophobic surface, the apparent contact angle exhibits oscillatory behavior (with a variation of about $4^{\circ}$ ) as soon as the contact line enters the hydrophilic section of the surface. Such instabilities could be the result of capillary wave propagation created at the receding wetting front on the surface[67]. 

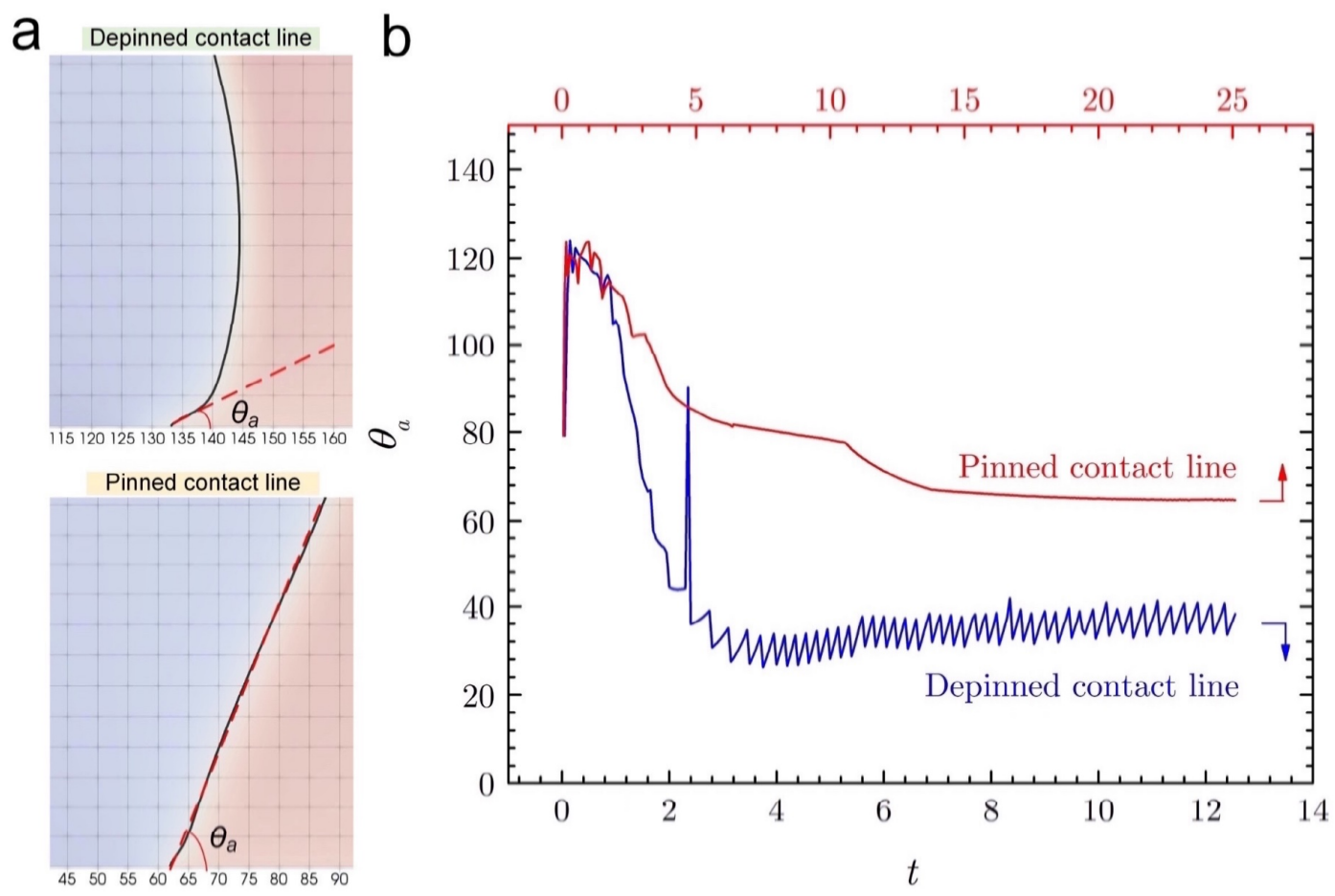

Fig. 8. Comparison of the apparent contact angles at the pinned and depinned contact lines. In (a) are shown examples of the contact-line regions close to the biphilic surface (with $\theta_{s, p h o}=130^{\circ}$ and $\left.\theta_{s, p h i}=10^{\circ}\right)$ under the DCL $(g=0.0002)$ and PCL $(g=0.0007)$ regimes, respectively. The vapor phase is represented in blue and the liquid phase in red. At $t=10.0$, the depinned contact line is being pulled by the rapidly growing bubble, which leads to bending of the bubble interface (in black, as defined by the density contour line at $\left.\left(\rho /+\rho_{v}\right) / 2\right)$ near the solid hydrophilic surface. The resulting apparent contact angle $\theta_{a}$ differs considerably from that of the pinned contact line, which extends along the stretched bubble interface from the border between the surfaces of opposing wettabilities to the outer region. In (b) the evolutions of $\theta_{a}$ across the wettability divide are plotted for these two cases, respectively. The results show more unstable behavior for the mobile contact line on the hydrophilic surface. Note that $\theta_{a}$ is calculated using two-point approximation near the wall $z=0$.

In Fig. 9 we present the representative values of $\theta_{a}$ (which are averaged over a period of at least $t=1.0$ once $\theta_{a}$ reaches steady state, as shown by the examples in Fig. 8(b)) under various wetting patterns and gravity conditions. Similar to Fig. 6, the distributions of $\theta_{a}$ over increasing $g$ appear to fall into two distinct groups that correspond incidentally to the DCL and PCL regimes. 
At relatively low $g$, the moving contact line on the hydrophilic surface seems to adopt an apparent contact angle that, albeit not a complete match, strongly correlates with the assigned $\theta_{s, p h i}$. The deviation of $\theta_{a}$ from $\theta_{s, p h i}$ becomes even less with growing $\theta_{s, p h i}$, which indicates improved local equilibrium as the dynamic effect weakens in the contact-line region. It should also be noted that $\theta_{a}$ exhibits no clear dependence on the gravity level, which corresponds to varying contact line speed (see Fig. 6). In other words, as we have argued above, the effective contact angle in question here should not be confused with the classic dynamic contact angle.

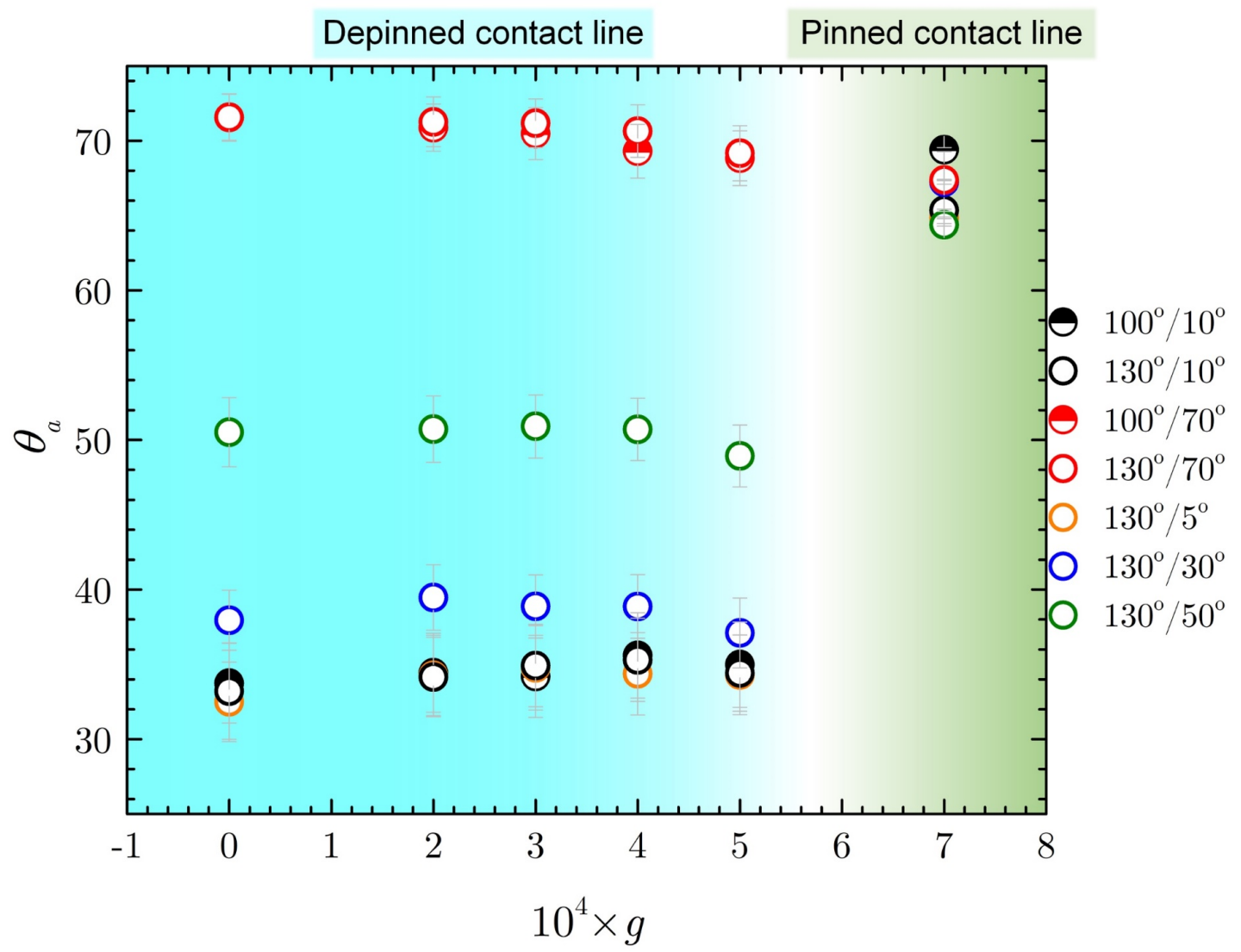

Fig. 9. Distributions of the steady-state apparent contact angle $\theta_{a}$ over different gravities, for various combinations of $\theta_{s, p h o}$ and $\theta_{s, p h i}$. Each data point represents averaged value of long-term $\theta_{a}$ over a period of at least $t=1.0$ and the error bars denote data spread. In accordance with the pinning state of contact line, the results can be clearly divided into two distinct groups. When the contact line is allowed to travel on the hydrophilic surface, $\theta_{a}$ appears to vary closely with $\theta_{s, p h i}$. On the other hand, when the contact line remains pinned at the interface between the surfaces of 
opposite wetting conditions, $\theta_{a}$ appears to take on a nearly universal value which is notably independent of either $\theta_{s, \text { pho }}$ or $\theta_{s, p h i}$.

When $g$ becomes sufficiently large, the contact line eventually gets pinned on the biphilic surface. Other than the considerably reduced mobility, the pinned contact line differs from the depinned contact line in another key aspect. That is, the apparent contact angles obtained at $g=0.0007$ seem to all fall around $\theta_{a}=66.4^{\circ} \pm 1.9^{\circ}$, irrespective of the surface wetting conditions of $\theta_{s, p h i}$ or $\theta_{s, p h o}$ (see Fig. 9). The nearly universal behavior signifies a delicate balance between the driving force of the (horizontal) bubble expansion and the pinning force at the contact line, which gives rise to the subsequent asymmetric bubble growth seen in Fig. 4. At the moment we lack a clear explanation for the interesting value of $\theta_{a}=66.4^{\circ}$, which could be subject to change under different fluid properties or characteristic sizes of the wetting inhomogeneities. More studies are needed to fully grasp the physical meaning of the constant pinned contact angle. In the following, we give a short discussion as regards the detailed mechanics of contact-line pinning.

\section{MECHANISM OF CONTACT-LINE PINNING}

Our next goal is to quantify the critical condition for contact-line pinning based on a simple theoretical model. Inspired by Kandlikar's seminal modeling work on boiling $\mathrm{CHF}[28]$ that notably included non-hydrodynamic influences such as surface wetting, we perform a similar force-balance analysis of bubble spreading on a biphilic surface that consists of opposing wettabilities.

Figure 10 depicts a bubble growing atop the hydrophobic section (in gray) of the biphilic surface, whose propagating three-phase contact line has just reached the border with the adjacent hydrophilic surface (in green). Under the pinned-contact-line assumption, as the present simulation has clearly indicated, the following bubble growth will continue at a fixed angle $\beta$ to the surface. Driving bubble expansion in the direction parallel to the surface is an evaporative momentum force $F_{m}$, which is given as (per unit length normal to the paper) 


$$
F_{m}=\left(\frac{q_{i}}{h_{l v}}\right)^{2} \frac{H_{b}}{\rho_{v}} .
$$

Here, $q_{i}$ represents the average heat flux per unit area across the bubble interface, which we presume is devoted entirely to local evaporation. Also, let $h_{l v}$ denote the latent heat of vaporization and $H_{b}$ the height of the bubble.

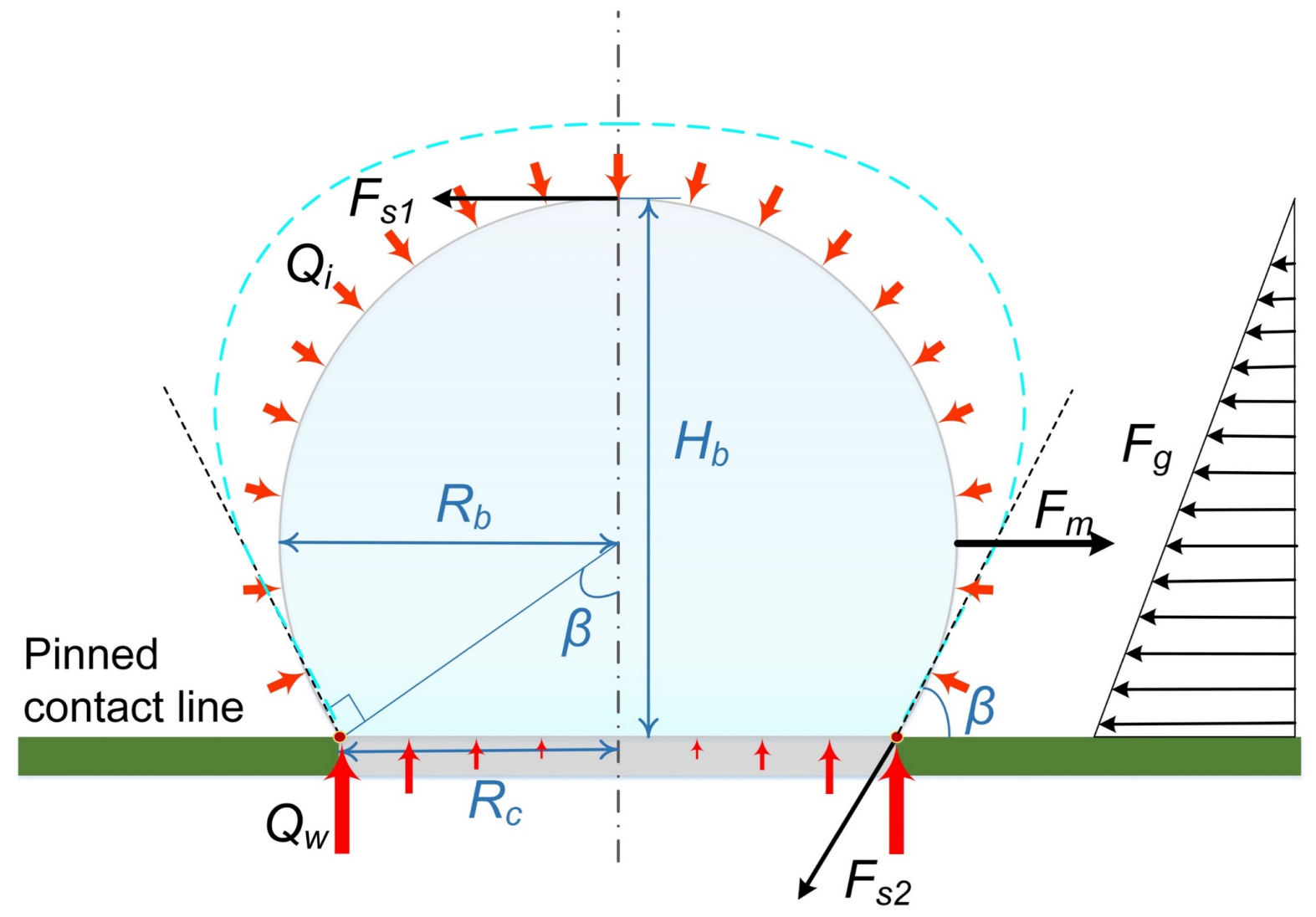

Fig. 10. A schematic illustration of the horizontal expansion of a bubble (of $R_{b}$ in width and $R_{c}$ in base radius) on a biphilic surface. Continuous bubble growth is driven by heat inputs $Q_{i}$ at the bubble interface and $Q_{w}$ beneath the bubble. When the driving force $F_{m}$ (due to the momentum variation during evaporation) roughly matches those resisting forces due to the surface tension effect, $F_{s 1}$ and $F_{s 2}$, and the hydrostatic pressure gradient $F_{g}$, the bubble tends to grow along a fixed angle $\beta$ to the surface. Moreover, the contact line will remain pinned at the border between the hydrophobic (in gray) and hydrophilic (in green) surfaces.

On the other hand, the forces resisting bubble spreading on the surface are comprised mainly of the surface tension forces and the excess pressure force (due to the hydrostatic pressure difference between the inside and outside of the bubble). Along 
the horizontal direction, the former is represented as (again, per unit length normal to the paper)

$$
F_{s}=F_{s 1}+F_{s 2} \cos \beta=\sigma(1+\cos \beta)
$$

whereas the latter reads

$$
F_{g}=\frac{1}{2} g\left(\rho_{l}-\rho_{v}\right) H_{b}^{2}
$$

We consider saturated bubble growth, of which the energy balance leads to

$$
Q_{i}+Q_{w}=\pi R_{b}^{2}(2-\cos \beta)(1+\cos \beta)^{2} \rho_{v} h_{l v} \dot{R}_{b}
$$

Note that $Q_{i}$ and $Q_{w}$ represent the total heat inflows per unit time through the bubble interface and from below the bubble (which, remarkably, peaks at the contact-line region[59]), respectively. Given the dominant contribution of $Q_{w}$ to bubble growth over that of $Q_{i}$, herein we assume without restrictions $Q_{w}=\xi_{Q} Q_{i}$, where the cofactor is taken to be $\xi_{Q}=4.0$ based on various bubble growth models[69]. Equation (25) then becomes

$$
q_{i}=\frac{1}{2 \xi_{Q}} \rho_{v} h_{l v}(2-\cos \beta)(1+\cos \beta) \dot{R}_{b}
$$

In deriving the above equation, use has been made of the total surface area of the bubble $A_{b}=2 \pi R_{b}^{2}(1+\cos \beta)$.

Displacing the contact line from the current affixed position requires the driving evaporative momentum force to exceed those resisting forces, which translates to $F_{m} \geq$ $F_{s}+F_{g}$. Substituting the definitions of the individual forces by virtue of Eqs. (22-24), we finally arrive at

$$
\begin{aligned}
\dot{R}_{b} \geq 2 \xi_{Q}\left[\frac{1}{2(2-\cos \beta)^{2}(1+\cos \beta) \sin \beta} \frac{g\left(\rho_{l}-\rho_{v}\right) R_{c}}{\rho_{v}}\right. \\
\left.+\frac{\sin \beta}{(2-\cos \beta)^{2}(1+\cos \beta)^{2}} \frac{\sigma}{R_{c} \rho_{v}}\right]^{\frac{1}{2}},
\end{aligned}
$$

where $R_{c}$ denotes the bubble base radius and is related to the bubble height by a simple geometric argument $H_{b}=(1+\cos \beta) R_{c} / \sin \beta$. The above equation describes the minimal bubble growth rate that is needed for the contact line to overcome the energy barrier 
due to the surface wetting inhomogeneities, below which the bubble spreading on the surface is expected to be severely hampered or even stalled. Furthermore, it would seem that pinning of contact-line is more likely to occur at greater $g$ 's, whereby evidently more robust bubble growth is required for free expansion parallel to the surface.

In the case of $R_{c}=60.0$ and $\beta \approx 66.4^{\circ}$ for a pinned contact line at $g=0.0007$, we calculate, using Eq. (27), the threshold bubble expansion rate for contact line pinning,

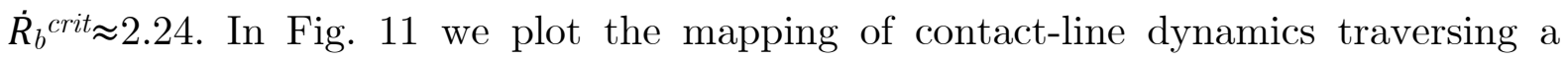
wettability divide, which includes results obtained under different biphilic combinations $\left(\theta_{s, p h i}\right.$ and $\left.\theta_{s, p h o}\right)$. The vertical axis denotes the average contact-line speed $\dot{R}_{c}$, whereas the horizontal axis denotes the corresponding bubble growth rate $\dot{R}_{b}$. It can be seen that a crossover from the DCL regime to the PCL regime occurs just around $\dot{R}_{b}{ }^{c r i t}$, in agreement with the theoretical prediction. The contact-line motion initially follows closely that of the bubble growth (exhibiting greater sensitivity to $\theta_{s, p h i}$ than to $\theta_{s, p h o}$, for that matter) until a drastic deceleration is triggered near $\dot{R}_{b}{ }^{\text {crit }}$, which leads eventually to demobilization of contact line.

Before concluding this section, a few remarks on the dramatic transition in the contact-line behavior are in order. Firstly, it should be mentioned that the data points in Fig. 11 are derived for different gravity levels, while only the critical bubble growth rate for the highest $g(=0.0007)$ is marked. Since $\dot{R}_{b}$ tends to increase with decreasing $g$ (as $\dot{R}_{b} \propto g^{-1}$, according to Fig. 6) and the opposite trend holds true for $\dot{R}_{b}{ }^{c r i t}$ (as $\dot{R}_{b}{ }^{c r i t} \propto g^{0.5}$, according to Eq. (27)), pinning of contact-line is therefore only feasible at sufficiently large $g$ where a dwindling $\dot{R}_{b}$ could possibly match a rising $\dot{R}_{b}{ }^{\text {crit }}$. Secondly, calculation of $\dot{R}_{b}$ crit (Eq. (27)) turns out to be sensitive to the parameter $\xi_{Q}$. The good agreement between the simulations and the model shown in Fig. 11 relies in no small part on the tentative value of $\xi_{Q}=4.0$. In fact, a more convincing comparison requires direct accurate measurement of $\xi_{Q}$ and expanded knowledge of heat flux partitioning in boiling on heterogeneous surfaces, which goes beyond the scope of the present study. Thirdly, the simulated transition in the contact-line interaction with the biphilic 
surface qualitatively agrees with our latest experimental observations of subatmospheric boiling characteristics[70]. The experiment revealed a strong correlation between the depinning of the contact line and the sudden acceleration of bubble growth at sufficiently reduced pressures, which matches the contact-line behavior depicted in Fig. 11. And lastly, the nature of the energy barrier to contactline motion, which is taken mainly as the surface tension force here, could be further explored on a molecular level. Recent MD simulations[71] have pointed to a critical characteristic size of chemical heterogeneities, below which the effect of contact-line pinning appears to vanish.

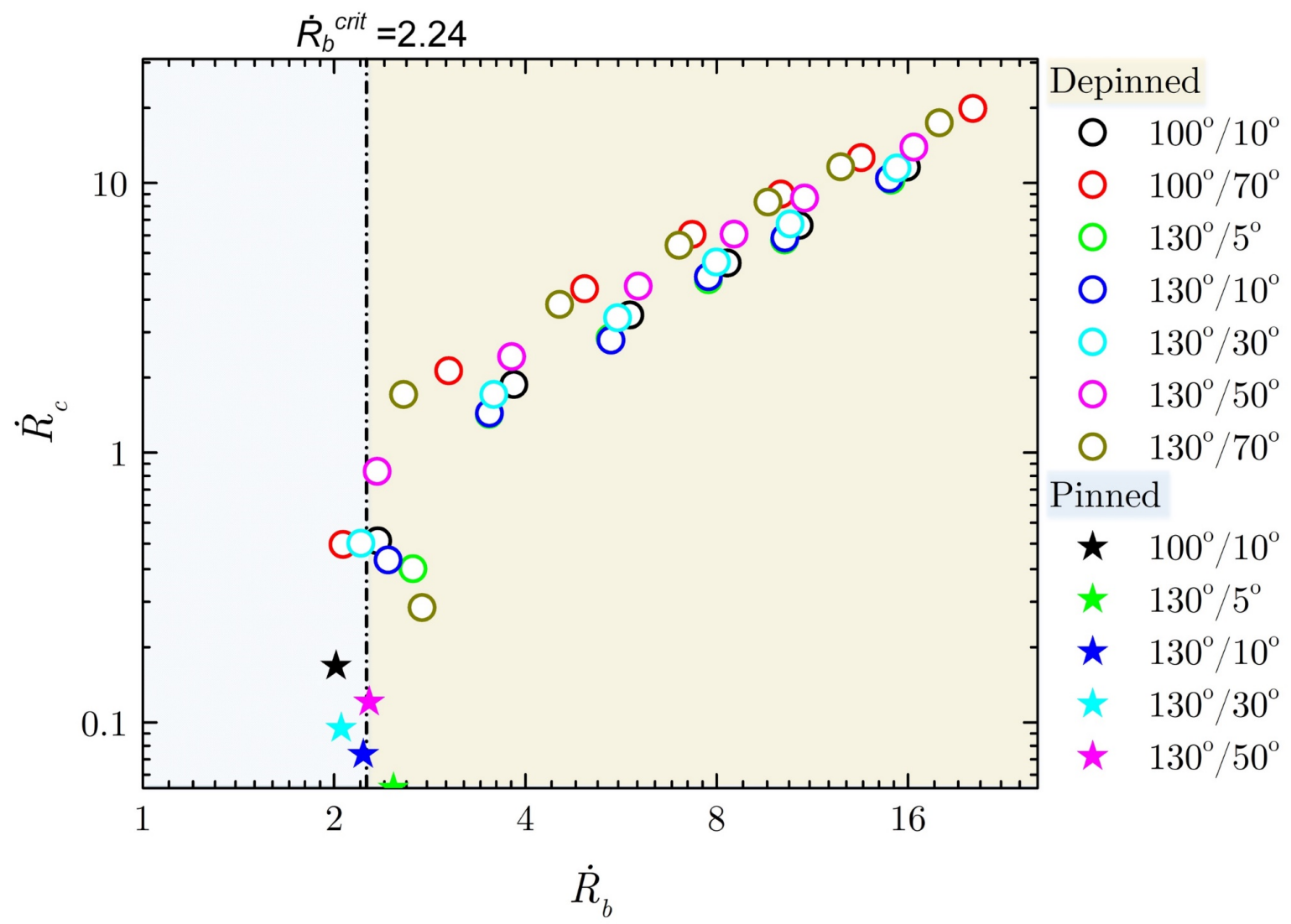

Fig. 11. Traversing contact-line speed $\dot{R}_{c}$ versus bubble growth rate $\dot{R}_{b}$. For all the patterns of surface biphilicity considered, the dramatic transition in the contact-line behavior between the regimes of contact-line depinning and contact-line pinning occurs around $\dot{R}_{b}^{\text {crit }}=2.24$, which is a value derived from the force-balance-based bubble spreading model. 


\section{CONCLUSIONS}

In this study, we have performed numerical simulations of contact-line motion on an isothermal biphilic surface by means of the diffuse-interface approach, where the liquid-vapor interface is treated as a continuous transition between the two phases and surface tension emerges naturally as the energy cost associated with the interfacial formation. The governing partial differential equations written for a single-component (water) system are discretized on an axisymmetric two-dimensional computational domain and solved by FemLego, a symbolic finite-element-based numerical solver.

The results reveal interesting contact-line behavior that can shed light on the puzzling observation of sudden boiling deterioration on biphilic surfaces at very low pressures in experiments. Following the fast expansion on the hydrophobic surface, the bubble evolution diverges depending on the bubble growth rate once the contact line reaches the edge of the adjacent hydrophilic surface. In the case of fast bubble expansion (at low gravity), the contact line motion is shown to experience a temporary hinderance at the wettability divide, which is due more to the enhanced surface wetting ahead, before becoming fully recovered on the hydrophilic surface. In sharp contrast to the case of depinned contact line, however, sufficiently weakened bubble growth (at high gravity) can result in a significant slowdown and even complete pinning of the contact line. Afterwards, the bubble growth seems to continue along a consistent apparent contact angle, which is independent of either the hydrophobic or the hydrophilic surfaces. The crossover between the regimes of mobile contact line and pinned contact line, which is well captured by mapping the contact-line dynamics on surfaces with different wetting conditions, can be largely explained on the basis of the force-balance model of bubble spreading across wetting inhomogeneities.

Exhibiting a great potential for manipulating bubble behavior, surface wettability engineering provides a genuinely promising solution to the challenging task of boiling heat transfer enhancement. Nevertheless, a unified description of contactline motion on biphilic surfaces is still lacking. It is our hope that the findings in this paper that are based on a simple wetting model can advance the fundamental 
understanding of how the contact line spreads on a wettability-patterned surface in the particularly vigorous events of boiling. Future research following the present work should expand on the interpretation of wetting behavior by including more complex physics such as contact line friction[72], which could foreseeably lead to new insights on controlled bubble generation and growth in boiling applications. 


\section{References}

[1] W. M. Rohsenow, "Boiling," Annu. Rev. Fluid Mech. 3, 211-236 (1971).

[2] V. K. Dhir, "Boiling heat transfer," Annu. Rev. Fluid Mech. 30, 365-401 (1998).

[3] G. Liang and I. Mudawar, "Review of pool boiling enhancement with additives and nanofluids," Int. J. Heat Mass Transf. 124, 423-453 (2018).

[4] G. Kjaerheim, "Heat transfer as limiting factor in water-cooled nuclear reactors," Nucl. Eng. Des. 21, 279-302 (1972).

[5] H. Gong, A. R. Khan, N. Erkan, L. Wang, and K. Okamoto, "Critical heat flux enhancement in downward-facing pool boiling with radiation induced surface activation effect," Int. J. Heat Mass Transf. 109, 93-102 (2017).

[6] I. Mudawar, "Recent Advances in High-Flux, Two-Phase Thermal Management," J. Therm. Sci. Eng. Appl. 5, 021012 (2013).

[7] Z. Li and S. G. Kandlikar, "Current status and future trends in data-center cooling technologies," Heat Transf. Eng. 36, 523-538 (2015).

[8] S. Nukiyama, "The maximum and minimum values of the heat Q transmitted from metal to boiling water under atmospheric pressure," Int. J. Heat Mass Transf. 9, 1419-1433 (1966).

[9] D. Attinger, C. Frankiewicz, A. R. Betz, T. M. Schutzius, R. Ganguly, A. Das, C.-J. Kim, and C. M. Megaridis, "Surface engineering for phase change heat transfer: A review," MRS Energy Sustain. 1, E4 (2014).

[10] R. Mallozzi, R. L. Judd, and N. Balakrishnan, "Investigation of randomness, overlap and the interaction of bubbles forming at adjacent nucleation sites in pool boiling," Int. J. Heat Mass Transf. 43, 3317-3330 (2000).

[11] V. K. Dhir, "Mechanistic Prediction of Nucleate Boiling Heat TransferAchievable or a Hopeless Task?," J. Heat Transfer 128, 1-12 (2006).

[12] H. Chi-Yeh and P. Griffith, "The mechanism of heat transfer in nucleate pool boiling-Part I," Int. J. Heat Mass Transf. 8, 887-904 (1965).

[13] B. . Mikic, W. . Rohsenow, and P. Griffith, "On bubble growth rates," Int. J. Heat Mass Transf. 13, 657-666 (1970). 
[14] N. Zuber, "Nucleate boiling. The region of isolated bubbles and the similarity with natural convection," Int. J. Heat Mass Transf. 6, 53-78 (1963).

[15] N. Zuber, "Hydrodynamic aspects of boiling heat transfer," Ph.D. thesis, Univ. California, Los Angeles (1959).

[16] S. J. D. van Stralen, R. Cole, W. M. Sluyter, and M. S. Sohal, "Bubble growth rates in nucleate boiling of water at subatmospheric pressures," Int. J. Heat Mass Transf. 18, 655-669 (1975).

[17] B. Bourdon, E. Bertrand, P. Di Marco, M. Marengo, R. Rioboo, and J. De Coninck, "Wettability influence on the onset temperature of pool boiling: Experimental evidence onto ultra-smooth surfaces," Adv. Colloid Interface Sci. 221, 34-40 (2015).

[18] T. . Theofanous, J. . Tu, A. . Dinh, and T. . Dinh, "The boiling crisis phenomenon," Exp. Therm. Fluid Sci. 26, 775-792 (2002).

[19] B. Shen, B. J. Suroto, S. Hirabayashi, M. Yamada, S. Hidaka, M. Kohno, K. Takahashi, and Y. Takata, "Bubble activation from a hydrophobic spot at "negative" surface superheats in subcooled boiling," Appl. Therm. Eng. 88, 230$236(2014)$.

[20] B. Shen, M. Yamada, S. Hidaka, J. Liu, J. Shiomi, G. Amberg, M. Do-Quang, M. Kohno, K. Takahashi, et al., "Early Onset of Nucleate Boiling on Gas-covered Biphilic Surfaces," Sci. Rep. 7, 2036 (2017).

[21] D. Lohse and X. Zhang, "Surface nanobubbles and nanodroplets," Rev. Mod. Phys. 87, 981-1035 (2015).

[22] J. S. Coursey and J. Kim, "Nanofluid boiling: The effect of surface wettability," Int. J. Heat Fluid Flow 29, 1577-1585 (2008).

[23] R. Chen, M. Lu, V. Srinivasan, Z. Wang, H. H. Cho, and A. Majumdar, "Nanowires for Enhanced Boiling Heat Transfer 2009," Nano Lett. 9, 548-553 (2009).

[24] J. A. Weibel, S. S. Kim, T. S. Fisher, and S. V. Garimella, "Carbon Nanotube Coatings for Enhanced Capillary-Fed Boiling from Porous Microstructures," 
Nanoscale Microscale Thermophys. Eng. 16, 1-17 (2012).

[25] A. Jaikumar and S. G. Kandlikar, "Ultra-high pool boiling performance and effect of channel width with selectively coated open microchannels," Int. J. Heat Mass Transf. 95, 795-805 (2016).

[26] S. Mori, N. Maruoka, and K. Okuyama, "Critical heat flux enhancement by a two-layer structured honeycomb porous plate in a saturated pool boiling of water," Int. J. Heat Mass Transf. 118, 429-438 (2018).

[27] S. J. Kim, I. C. Bang, J. Buongiorno, and L. W. Hu, "Surface wettability change during pool boiling of nanofluids and its effect on critical heat flux," Int. J. Heat Mass Transf. 50, 4105-4116 (2007).

[28] S. G. Kandlikar, "A Theoretical Model to Predict Pool Boiling CHF Incorporating Effects of Contact Angle and Orientation," J. Heat Transfer 123, 1071 (2001).

[29] M. M. Rahman, E. Ölçeroglu, and M. McCarthy, "Role of wickability on the critical heat flux of structured superhydrophilic surfaces," Langmuir 30, 1122511234 (2014).

[30] M. Ha and S. Graham, "Pool boiling characteristics and critical heat flux mechanisms of microporous surfaces and enhancement through structural modification," Appl. Phys. Lett. 111, 091601 (2017).

[31] N. S. Dhillon, J. Buongiorno, and K. K. Varanasi, "Critical heat flux maxima during boiling crisis on textured surfaces," Nat. Commun. 6, 8247 (2015).

[32] T. P. Allred, J. A. Weibel, and S. V. Garimella, "Enabling Highly Effective Boiling from Superhydrophobic Surfaces," Phys. Rev. Lett. 120, 174501 (2018).

[33] T. P. . Allred, J. A. . Weibel, and S. V. Garimella, "The petal effect of parahydrophobic surfaces offers low receding contact angles that promote effective boiling," Int. J. Heat Mass Transf. 135, 403-412 (2019).

[34] C. Frankiewicz and D. Attinger, "On Temporal Biphilicity: Definition, Relevance, and Technical Implementation in Boiling Heat Transfer," J. Heat Transfer 139, $111511(2017)$. 
[35] A. Sur, Y. Lu, C. Pascente, P. Ruchhoeft, and D. Liu, "Pool boiling heat transfer enhancement with electrowetting," Int. J. Heat Mass Transf. 120, 202-217 (2018).

[36] A. R. Betz, J. Xu, H. Qiu, and D. Attinger, "Do surfaces with mixed hydrophilic and hydrophobic areas enhance pool boiling?," Appl. Phys. Lett. 97, 141909 (2010).

[37] M. M. Rahman, J. Pollack, and M. McCarthy, "Increasing Boiling Heat Transfer using Low Conductivity Materials," Sci. Rep. 5, 13145 (2015).

[38] A. Kalani and S. G. Kandlikar, "Enhanced Pool Boiling With Ethanol at Subatmospheric Pressures for Electronics Cooling," J. Heat Transfer 135, 111002 (2013).

[39] M. Yamada, B. Shen, T. Imamura, S. Hidaka, M. Kohno, K. Takahashi, and Y. Takata, "Enhancement of boiling heat transfer under sub-atmospheric pressures using biphilic surfaces," Int. J. Heat Mass Transf. 115, 753-762 (2017).

[40] B. Shen, M. Yamada, T. Mine, S. Hidaka, M. Kohno, K. Takahashi, and Y. Takata, "Depinning of bubble contact line on a biphilic surface in subatmospheric boiling: Revisiting the theories of bubble departure," Int. J. Heat Mass Transf. 126, 715-720 (2018).

[41] Q. Li, P. Zhou, and H. J. Yan, "Pinning-Depinning Mechanism of the Contact Line during Evaporation on Chemically Patterned Surfaces: A Lattice Boltzmann Study," Langmuir 32, 9389-9396 (2016).

[42] J. Zhang, F. Müller-Plathe, and F. Leroy, "Pinning of the Contact Line during Evaporation on Heterogeneous Surfaces: Slowdown or Temporary Immobilization? Insights from a Nanoscale Study," Langmuir 31, 7544-7552 $(2015)$.

[43] N. Anantharaju, M. Panchagnula, and S. Neti, "Evaporating drops on patterned surfaces: Transition from pinned to moving triple line," J. Colloid Interface Sci. 337, 176-182 (2009).

[44] S. Varagnolo, D. Ferraro, P. Fantinel, M. Pierno, G. Mistura, G. Amati, L. 
Biferale, and M. Sbragaglia, "Stick-Slip Sliding of Water Drops on Chemically Heterogeneous Surfaces," Phys. Rev. Lett. 111, 066101 (2013).

[45] X. P. Wang, T. Qian, and P. Sheng, "Moving contact line on chemically patterned surfaces," J. Fluid Mech. 605, 59-78 (2008).

[46] A. Jaikumar and S. G. Kandlikar, "Coupled Motion of Contact Line on Nanoscale Chemically Heterogeneous Surfaces for Improved Bubble Dynamics in Boiling," Sci. Rep. 7, 15691 (2017).

[47] D. M. Anderson, G. B. McFadden, and A. A. Wheeler, "DIFFUSE-INTERFACE METHODS IN FLUID MECHANICS," Annu. Rev. Fluid Mech. 30, 139-165 (1998).

[48] B. Shen, J. Liu, J. Shiomi, G. Amberg, M. Do-Quang, M. Kohno, K. Takahashi, and Y. Takata, "Effect of dissolved gas on bubble growth on a biphilic surface: A diffuse-interface simulation approach," Int. J. Heat Mass Transf. 126, 816$829(2018)$.

[49] C. R. Kharangate and I. Mudawar, "Review of computational studies on boiling and condensation," Int. J. Heat Mass Transf. 108, 1164-1196 (2017).

[50] Lord Rayleigh, "XX. On the theory of surface forces. - II. Compressible fluids," London, Edinburgh, Dublin Philos. Mag. J. Sci. 33, 209-220 (1892).

[51] Van der Waals J. D., "The thermodynamic theory capillarity under the hypothesis of a continuous variation of density. Verhandel. Konink. Akad. Weten. Amsterdam 1, 8 (1893); translation published by Rowlinson J. S.," J. Stat. Phys. 20, 197-200 (1979).

[52] M. Sussman, A. S. Almgren, J. B. Colella, L. H. Howell, and M. L. Welcome, "An adaptive level set approach for incompressible two phase flows," J. Comput. Phys. 148, 81-124 (1999).

[53] Y. Sato and B. Niceno, "Pool boiling simulation using an interface tracking method: From nucleate boiling to film boiling regime through critical heat flux," Int. J. Heat Mass Transf. 125, 876-890 (2018).

[54] C. Huh and L. . Scriven, "Hydrodynamic model of steady movement of a 
solid/liquid/fluid contact line," J. Colloid Interface Sci. 35, 85-101 (1971).

[55] W. Ren and W. E, "Boundary conditions for the moving contact line problem," Phys. Fluids 19, 022101 (2007).

[56] X. Xu and T. Qian, "Contact line motion in confined liquid-gas systems: Slip versus phase transition," J. Chem. Phys. 133, 204704 (2010).

[57] A. Carlson, G. Bellani, and G. Amberg, "Universality in dynamic wetting dominated by contact-line friction," Phys. Rev. E 85, 045302 (2012).

[58] J. Liu, M. Do-Quang, and G. Amberg, "Thermohydrodynamics of boiling in binary compressible fluids," Phys. Rev. E 92, 043017 (2015).

[59] T. Laurila, A. Carlson, M. Do-Quang, T. Ala-Nissila, and G. Amberg, "Thermohydrodynamics of boiling in a van der Waals fluid," Phys. Rev. E 85, $026320(2012)$.

[60] J. W. Cahn and J. E. Hilliard, "Free energy of a nonuniform system. I. Interfacial free energy," J. Chem. Phys. 28, 258-267 (1958).

[61] A. Onuki, "Dynamic van der Waals theory," Phys. Rev. E 75, 036304 (2007).

[62] X. Xu and T. Qian, "Single-bubble dynamics in pool boiling of one-component fluids," Phys. Rev. E 89, 063002 (2014).

[63] G. Amberg, R. Tönhardt, and C. Winkler, "Finite element simulations using symbolic computing," Math. Comput. Simul. 49, 257-274 (1999).

[64] H. Ding and P. D. M. Spelt, "Wetting condition in diffuse interface simulations of contact line motion," Phys. Rev. E 75, 046708 (2007).

[65] R. Siegel and E. G. Keshock, "Effects of reduced gravity on nucleate boiling bubble dynamics in saturated water," AIChE J. 10, 509-517 (1964).

[66] J. Zhang, H. Huang, and X. Y. Lu, "Pinning-Depinning Mechanism of the Contact Line during Evaporation of Nanodroplets on Heated Heterogeneous Surfaces: A Molecular Dynamics Simulation," research-article, Langmuir 35, 6356-6366 (2019).

[67] A. Carlson, M. Do-Quang, and G. Amberg, "Dissipation in rapid dynamic wetting," J. Fluid Mech. 682, 213-240 (2011). 
[68] T. D. Blake, "The physics of moving wetting lines," J. Colloid Interface Sci. 299, $1-13$ (2006).

[69] S. Raj, M. Pathak, and M. K. Khan, "An analytical model for predicting growth rate and departure diameter of a bubble in subcooled flow boiling," Int. J. Heat Mass Transf. 109, 470-481 (2017).

[70] B. Shen, T. Mine, N. Iwata, S. Hidaka, K. Takahashi, and Y. Takata, "Deterioration of boiling heat transfer on biphilic surfaces under very low pressures," Exp. Therm. Fluid Sci. 113, 110026 (2020).

[71] F. C. Wang and H. A. Wu, "Pinning and depinning mechanism of the contact line during evaporation of nano-droplets sessile on textured surfaces," Soft Matter 9, 5703-5709 (2013).

[72] P. Johansson and B. Hess, "Molecular origin of contact line friction in dynamic wetting," Phys. Rev. Fluids 3, 074201 (2018). 